\title{
Jet Heated X-ray Filament in the Centaurus A Northern Middle Radio Lobe
}

\author{
R. P. Kraft ${ }^{1}$, W. R. Forman ${ }^{1}$,M. J. Hardcastle ${ }^{2}$, M. Birkinshaw ${ }^{3}$, J. H. Croston ${ }^{2}$,C. Jones ${ }^{1}$, \\ P. E. J. Nulsen ${ }^{1}$, D. M. Worrall ${ }^{3}$, S. S. Murray ${ }^{1}$
}

\begin{abstract}
We present results from a $40 \mathrm{ks} X M M$-Newton observation of the X-ray filament coincident with the southeast edge of the Centaurus A Northern Middle Radio Lobe (NML). We find that the X-ray filament consists of five spatially resolved X-ray knots embedded in a continuous diffuse bridge. The spectrum of each knot is well fitted by a thermal model with temperatures ranging from 0.3-0.7 keV and subsolar elemental abundances. In four of the five knots, nonthermal models are a poor fit to the spectra, conclusively ruling out synchrotron or IC/CMB mechanisms for their emission. The internal pressures of the knots exceed that of the ambient ISM or the equipartition pressure of the NML by more than an order of magnitude, demonstrating that they must be short lived $\left(\sim 3 \times 10^{6} \mathrm{yrs}\right)$. Based on energetic arguments, it is implausible that these knots have been ionized by the beamed flux from the active galactic nucleus of Cen A or that they have been shock-heated by supersonic inflation of the NML. In our view, the most viable scenario for the origin of the X-ray knots is that they are the result of cold gas shock heated by a direct interaction with the jet. The lifetimes of the X-ray knots in the NML are roughly the same as the age of the strong shock around the SW inner lobe, suggesting they were created in the same AGN outburst. The most plausible model of the NML is that it is a bubble from a previous outburst that is being re-energized by the current outburst. We also report the discovery of a large scale (at least $35 \mathrm{kpc}$ radius) gas halo around Cen A.
\end{abstract}

Subject headings: galaxies: individual (Centaurus A, NGC 5128) - X-rays: galaxies - hydrodynamics - galaxies: jets

\footnotetext{
${ }^{1}$ Harvard/Smithsonian Center for Astrophysics, 60 Garden St., MS-67, Cambridge, MA 02138

${ }^{2}$ University of Hertfordshire, School of Physics, Astronomy, and Mathematics, Hatfield AL10 9AB, UK

${ }^{3}$ University of Bristol, Department of Physics, Tyndall Avenue, Bristol BS8 ITL, UK
} 


\section{Introduction}

Twin jets launched from the active nuclei of radio galaxies provide an efficient mechanism for transferring gravitational energy of gas infalling onto a supermassive black hole (SMBH) back into thermal energy of the ambient gas out to distances hundreds of kpc from the nuclei. The conventional paradigm suggests that the synchrotron-emitting radio lobes of radio galaxies contain relativistic plasma supplied by jets. In the early stage, the momentum of the jets dominates the dynamical evolution. Depending on the mechanical power of the jet and richness of the ambient environment, the jet will initially drive a strong shock into the ambient gas. As the bubble becomes large and the radio galaxy evolves, the advance speed of the jet head decreases and the energy/pressure of the bubble determines the dynamical evolution. Once the energy supply from the active nucleus ceases, the bubble will come into rough pressure equilibrium with the ambient gas and its dynamics will largely be driven by buoyancy in the stratified atmosphere.

Centaurus $\mathrm{A}$ is the nearest radio galaxy to the Milky Way $\left(d=3.7 \mathrm{Mpc}, 1^{\prime}=1.076 \mathrm{kpc}\right)$, and exhibits a complex morphology on spatial scales from milliarcseconds to degrees. A montage of the radio emission from Cen A on various spatial scales is shown in Figure 1 of Morganti et al. (1999). VLBI observations of Cen A (spatial scales of tens of milliarcseconds) detected the bright AGN, a forward jet, and a counter-jet. Based on measurements of the sidedness of the VLBI jets, the jet velocity is estimated to be $\sim 0.3 c$. On arcminute scales, Burns, Feigelson, \& Schreier (1983) detected a one-sided radio jet extending $\sim 4^{\prime}$ to the northeast of the nucleus opening into a radio lobe (the NE inner lobe, or simply NE lobe). A second lobe of similar size and radio brightness was detected to the southwest (the SW lobe) with no obvious radio counter-jet feeding it. Burns, Feigelson, \& Schreier (1983) found only a few spatially resolved radio knots embedded in the plasma of the SW lobe.

Chandra and XMM-Newton observations of the inner jet and radio lobes show significant differences between the NE lobe and the SW lobe. The dominant X-ray emission mechanism from the inner jet is synchrotron radiation emanating from $\sim 30$ knots embedded in diffuse emission. There are significant spatial offsets between the X-ray and radio peaks of the inner jet (Kraft et al. 2002), and proper motions of some radio knots show velocities of $\sim 0.5 c$ (Hardcastle et al. 2003). Deep Chandra observations find extended diffuse emission associated with the radio knots in the SW lobe, conclusively demonstrating that this is the counter jet (Hardcastle et al. 2007). A shell of shock-heated gas and X-ray

synchrotron emitting ultra-relativistic electrons surrounds the SW lobe (Kraft et al. 2003, 2007; Croston et al. 2009). The temperature and density of the gas in the shell is several times that of the ambient medium. Thus, the inflation of the SW lobe is driving a strong shock into the ISM. Surprisingly, there is no corresponding shell of shock-heated gas around 
the NE lobe. There are a few filaments of X-ray emission around the NE lobe that may or may not be shock-heated gas, but the emissivity of these features is roughly two orders of magnitudes lower than the shell around the SW lobe (Kraft et al. 2007).

A much larger, lower surface brightness radio lobe, called the Northern Middle Lobe (NML), lies to the northeast beyond the NE (inner) lobe, $\sim 20-30 \mathrm{kpc}$ (roughly 20'-30') from the active nucleus (Junkes et al. 1993). This lobe is connected to the NE lobe by the large scale jet (LSJ) (Morganti et al. 1999). Whether the LSJ is actually a jet in the traditional sense that it represents a continuous channel for the transfer of energy and momentum in a collimated flow is unclear based on its radio morphology. Several sets of optical emission line filaments are associated with the NML and LSJ. The inner optical filament lies $\sim 2^{\prime}$ to the southeast of the LSJ (Blanco et al. 1975) and is coincident with weak X-ray emission (Evans \& Koratkar 2004). The outer filaments lie in the interior of the NML in projection, roughly 15-20' from the nucleus (Graham 1998). Several regions of star formation also lie in the interior of the NML in projection, perhaps the best known examples of jet-induced star formation (Mould et al. 2000). An HI cloud lies at the base of the NML just north of where the LSJ enters the lobe (Oosterloo \& Morganti 2005). Interestingly, there is no radio feature corresponding to the NML lying beyond the SW (inner) lobe. It has been proposed that the NML is either a buoyant bubble from a previous epoch of nuclear activity (Saxton. Sutherland, \& Bicknell 2003) or that the NE inner radio lobe has burst (Morganti et al. 1999). In the first scenario, the age of the NML would be on the order of several hundred Myrs, in the latter its age would be roughly the age of the inner radio lobes - only several Myrs.

Feigelson et al. (1981) reported the discovery of an X-ray filament along the southeast edge of the NML from an Einstein IPC observation. This feature was also detected in EXOSAT (Morini et al. 1989), ASCA (Morganti et al. 1999), and an unpublished archival ROSAT PSPC observation. Feigelson et al. (1981) argued that this feature was probably from hot gas, and rejected both synchrotron emission from a population of ultra-relativistic electrons and inverse-Compton scattering of CMB photons as viable possibilities. The synchrotron scenario would require an unusual X-ray to radio flux ratio, very different from that seen in FR I jets in which the X-ray emission is believed to be synchrotron. Likewise, the inverse-Compton scattering of the microwave background photons (IC/CMB) hypothesis would require a large $\left(B_{N M L}<B_{e q} / 10\right)$ departure from equipartition, much larger than is seen in a sample of radio lobes observed with Chandra and XMM-Newton (Croston et al. 2005). In the IC/CMB scenario, the morphology of the X-ray emission must match the distribution of the low energy electrons and therefore be somewhat similar to the radio lobe (which depends on both the electron distribution and magnetic field strength). The morphological dissimilarities between the emission in the two bands offer a second strong argument 
against the IC/CMB scenario. The X-ray filament is associated with a region of lower radio polarization in the NML, adding support to the thermal gas hypothesis (Morganti et al. 1999). Saxton, Sutherland, \& Bicknell (2003) have modeled the NML as a buoyant bubble and identify the X-ray filament as the thermal gas trunk of material that has been drawn up behind the bubble from the central regions of the host galaxy.

Optically Cen A is crossed by a dark dust band that is believed to be the result of a merger with a small spiral galaxy similar to M33 several hundred million years ago (Malin et al. 1983; Israel 1998). We note that there is considerable debate in the literature as to the age of the merger, with low estimates on the order of 100 Myrs (Quillen et al. 1992) and high estimates of several Gyrs (Soria et al. 1996). The low end of this range is roughly consistent with the age of the NML if it is a buoyant bubble. The merger certainly induced large scale gas motions that, depending on the time since the merger and the viscosity of the ISM, may or may not have dissipated. Such gas motions may play an important role in shaping the large scale morphology of the radio source. It is possible that the accretion of cold gas by the central SMBH provided by the spiral is fueling the current epoch of nuclear activity. The merger may well have fueled the earlier epochs of nuclear activity that created the radio structures on tens of arcminutes and degree scales.

In this paper, we present results from a $40 \mathrm{ks} X M M-N e w t o n$ observation of the X-ray filament associated with the Cen A NML. The primary science objective of this observation was to determine the nature of the X-ray emission from the NML filament and to determine whether the NML is produced by the current nuclear outburst or is a remnant of an earlier one. This paper is organized as follows. Section 2 contains a brief summary of the data. Analysis is presented in section 3, followed by an interpretation of our results in Section 4. Section 5 contains a brief summary and conclusion. We adopt a distance of $3.7 \mathrm{Mpc}$ to Cen A, which is the mean of distance measures from five independent indicators (TRGB, Mira variables, SBF, PNLF, Cepheids, summarized in Section 6 of Ferrarese et al. (2007)). At this distance, $1^{\prime \prime}=17.9 \mathrm{pc}$ and $1^{\prime}=1.076 \mathrm{kpc}$. All uncertainties are at $90 \%$ confidence for one parameter of interest unless otherwise stated, and all coordinates are J2000. All spectral fits include absorption $\left(N_{H}=8.41 \times 10^{20} \mathrm{~cm}^{-2}\right)$ by foreground gas in our Galaxy (Dickey \& Lockman 1990).

\section{Data Analysis}

The Cen A NML was observed for $\sim 40$ ks with XMM-Newton on February 2, 2006 (OBSID 0302070101, revolution 1127) with the Medium filter inserted. The MOS and PN event files were filtered using a strict criterion for highest energy resolution. All events in 
the MOS event files with FLAG $>0$ and PATTERN $>12$ were excluded. All events in the PN event file with FLAG $>0$ and PATTERN $>4$ were excluded. Light curves were made for all three event files in the 5.0-10.0 keV band and periods when the background exceeded the mean value by more than $3 \sigma$ were removed. The time removed due to background flaring was relatively small. Flare filtering left 38477 s, $38126 \mathrm{~s}$, and $26540 \mathrm{~s}$ of good time in the MOS1, MOS2, and PN cameras, respectively. All point sources visible by eye were excluded from our analysis.

Data from three other X-ray observations are used in the analysis presented in this paper. The central region of Cen A was observed with XMM-Newton twice (OBSIDs 0093650201 and 0093650301) for observation times of $36055 \mathrm{~s}, 36045 \mathrm{~s}$, and $24758 \mathrm{~s}$ in the MOS1, MOS2 and PN cameras, respectively. Results from these observations were originally published in Kraft et al. (2003) and Evans et al. (2004). The southern part of the Cen A NML is also contained within the FOV of one Chandra/ACIS-I observation (OBSID 7800 - $100 \mathrm{ks}$ ). Several of the knots of the NML are located on the S2 chip in this observation, $\sim 15^{\prime}$ off axis. Since the NML is so far off axis, we use these data only to confirm the measured temperatures of several of the fainter knots (N3, N4, and N5) at the southwestern boundary of the filament. Data from an archival ROSAT PSPC observation $(\sim 13.5 \mathrm{ks})$ is used to constrain the nature of the large scale gas halo. Finally, we use previously published VLA (Burns, Feigelson, \& Schreier 1983; Hardcastle et al. 2003) and ATCA (Morganti et al. 1999) radio data taken from public archives for comparison with our X-ray data.

\section{Analysis}

The large-scale spatial relationship between the X-ray filament of the NML, the radio components of the NML and inner lobes, and the X-ray gas of the central regions of Cen $\mathrm{A}$ is shown in Figure1, This is a Gaussian smoothed, exposure corrected MOS (1+2) image in the 0.3-1.0 keV band of all the XMM-Newton data on Cen A. The inner jet and inner radio lobes are shown by the green contours, the NML with the yellow contours. The X-ray filament lies along the southeastern boundary of the NML. Approximate positions are indicated for several other features of interest, including the HI cloud (red) and the inner and outer optical filaments (cyan) are also shown. There are clear peaks in the radio emission between the pairs of X-ray knots N1/N2, N2/N3, and N3/N4. The same image is shown in Figure 2 without the radio contours or other overlays to more clearly distinguish the X-ray filament, the large scale diffuse emission (the light blue), and the background level (the black).

An unsmoothed X-ray image (MOS1+MOS2 co-added, 4" per pixel) of the Cen A NML in the $0.5-2.0 \mathrm{keV}$ band is shown in Figure 3. The X-ray filament of the NML is 
composed of five distinct X-ray knots of emission (labeled N1 through N5 in Figure 3) with a bridge of diffuse emission connecting all the knots and extending to the east beyond knot N1. The point sources have been retained in this image, clearly showing that all five knots are spatially extended at the resolution of XMM-Newton. Figure 4 contains a smoothed (Gaussian - $\sigma=24^{\prime \prime}$ kernel), exposure corrected (MOS1+MOS2 coadded) image in the 0.5-2.0 $\mathrm{keV}$ band of the NML X-ray filament. The three knots closest to the nucleus (N3, N4, and N5) are also contained within the S2 chip of an ACIS-I observation of Cen A as shown in Figure 5. The NML is $\sim 15^{\prime}$ off-axis in this observation, so the PSF is fairly large, but the three knots can clearly be seen, as well as the diffuse emission between the knots. Another diffuse feature lies $2.5^{\prime}$ to the NW of knot N3, perhaps related to the rest of the filament. Two bright point sources are located at the SW edge of the chip, indicative of the size of the $\mathrm{PSF}$ in this observation. Interestingly, the X-ray knots are anti-coincident with the radio knots of the NML. This can be seen most clearly in the radio map with X-ray contours overlaid in Figure 6.

We fitted thermal and power-law models to the spectra of each of the five knots. Circular extraction regions were used to include all of the emission for each of the five knots. The positions and sizes of these regions are given in Table 1. Background was determined from two different local regions, one just to the northwest of the X-ray filament and a second along the periphery of the field of view. The results are insensitive to which background region is used in the fits. We quote results using the first background region only. Data from the MOS1, MOS2, and PN cameras were fitted simultaneously.

For each knot, the spectra were fitted with two models: an absorbed power law spectrum and an absorbed thermal (APEC) model with the elemental abundance as a free parameter. In all cases the absorption was held fixed at the Galactic value. Allowing this parameter to vary freely made no statistically significant difference in any of the fits, and the statistical uncertainty in this value was always consistent with the Galactic value. For knots N1 through $\mathrm{N} 4$, the power law model can be ruled out at high (>>99\%) confidence, thus strongly disfavoring a non-thermal (synchrotron or IC/CMB) origin for the X-ray emission. In all cases the thermal model provided an acceptable fit to the spectrum. A summary of the best fit temperatures and abundances is shown in Table 1.

\subsection{The Structure of Knot N1}

There is considerable substructure present in knot N1 which can be seen more clearly in Figure 7. The bridge structure connecting N1 to N2 lies along the east/west axis and extends beyond N1 to the east. The peak of the X-ray emission lies roughly $1.2^{\prime}$ south of 
where this bridge runs through the knot. In fact, the emission from all of the knots generally lies to the south and east of the thin bridge that connects them. The bridge extends to the east of knot N1 past the edge of the radio lobe (see Figure 11). This suggests that the radio emission from the NML visible in the ATCA $1.5 \mathrm{GHz}$ maps does not define the full extent of the NML.

\subsection{Knot N5}

The best fit temperature of knot N5 is considerably higher $(\sim 1 \mathrm{keV}$ in the $X M M$-Newton data, and $>2.5 \mathrm{keV}$ in the Chandra data) than that of the other four knots. In our favored model described below, in which the X-ray knots represent jet-cloud interactions, the higher temperature of N5 could suggest that the ram pressure of the jet impacting this cloud is somewhat higher than further along into the NML. It is also possible, however, that this feature is somehow different from the others or even unrelated to the NML. Knot N5 is clearly extended in both data sets, and is connected to knot N4 by the bridge, supporting the idea that this feature is part of the X-ray structure associated with the NML. On the other hand, its spectrum can be well fitted by an absorbed power-law model (photon index $1.8 \pm 0.3$ ), consistent with the synchrotron or IC/CMB emission mechanisms. We proceed with the assumption that this feature is thermal gas related to the rest of the X-ray filament. Our results and general conclusions on the X-ray features as a whole are unaffected if some of all of the flux from this feature is due to non-thermal emission or (perhaps) unresolved point sources.

\subsection{Bridge structure connecting knots}

We attempted to fit the XMM-Newton spectra of the thin bridge connecting the 5 bright X-ray knots, but the count rate was too low and the background too high to obtain a meaningful result. We did fit the emission between the pairs N3/N4 and N4/N5 in the Chandra data using the off-axis S2 CCD. The data were combined into one spectrum to maximize signal to noise, and a local region on the S2 chip away from the bridge was used to estimate background. The spectrum is well described by an absorbed thermal model with a temperature of $0.72 \pm 0.06 \mathrm{keV}$. The abundance is poorly constrained. A single power-law model can be rejected at high significance. The spectrum is entirely consistent with that of the bright knots confirming that they are of similar origin. 


\subsection{Hot ISM of Cen A}

There is clearly an asymmetry in the diffuse X-ray emission on either side (NW/SE) of the X-ray filament (see Figures 1 and 2). We interpret this excess diffuse emission to the $\mathrm{SE}$ of the filament as the hot ISM of Cen A. There is a region of enhanced X-ray surface brightness (the light blue in Figure 2) that extends more or less continuously toward the nucleus. This is the extended gas corona of Cen A. In fact, diffuse X-ray emission fills the XMM-Newton FOV of both observation and therefore extends more than $30 \mathrm{kpc}\left(30^{\prime}\right)$ from the active nucleus. The actual extent of the ISM is unclear, an observation over a larger FOV is required to map its extent. We note that the cavity filled by the radio plasma of the NML appears to be devoid of this X-ray emission (the black in Figure 2). The inflation of the NML must have evacuated this cavity; the lack of X-ray emission demonstrates that the radio plasma and thermal gas are not well mixed. The visibility of the X-ray depression confirms that the lobe lies fairly close to the plane of the sky. If the lobe were far from the plane of the sky, the evacuated cavity would not make a noticeable decrement in the X-ray emission. There is considerable asymmetric temperature and surface brightness structure in this emission in both the XMM-Newton and Chandra observations. The general structure of the diffuse emission is also seen in the ROSAT PSPC observation of Cen A. Details of this structure will be presented in a future publication (Kraft et al. in preparation). For the purposes of this paper, we only need to obtain a rough estimate of the gas density and pressure to assist us in our interpretation of the emission coincident with the NML. We note that this extended halo was treated as background in Kraft et al. (2003). Density estimates in the central regions of the galaxy are still approximately corrected, but any extrapolation of

the surface brightness profiles shown in Kraft et al. (2003) to more than several arcminutes from the nucleus do not account for this extended halo as it was subtracted as background.

The surface brightness profile of the gas in a $10^{\circ}$ wedge in the $0.3-1.0 \mathrm{keV}$ band is shown in Figure 8, This profile was taken from a smoothed, exposure corrected image (MO1+MOS2 combined), and the wedge lies just below (south) of the large scale jet and NML. The vertex of the wedge is at the nucleus. Background was determined from a distant region to the NW of the X-ray filament. We assume that the radio lobe completely evacuates a cavity in the ISM, and that the integrated path length through the gas outside of the lobe along our line of sight is small. Some fraction of the emission in this region may be due to gas in Cen A, but the background level at the interior of the lobe is roughly consistent with XMM-Newton dark sky background level. This could make a small difference to our derived density, but since the density goes as the square root of the surface brightness, the potential error is smaller than the uncertainty in the density due to the abundance. We determined the temperature of the hot coronal gas by fitting the spectrum of a circular region $275^{\prime \prime}$ in radius at the base of the NML in the XMM-Newton data. We find a temperature of $0.35 \pm 0.04 \mathrm{keV}$ 
with low abundance. We model the density profile as a sphere of uniform density with a radius of $35 \mathrm{kpc}$ (the results are insensitive to this choice). This model is obviously only an approximation that facilitates order of magnitude estimates of density and pressure. Such a flat profile out to large radii (20 kpc or more) is atypical for isolated early-type galaxies like Cen A and suggests that the large scale corona is still dynamically evolving either from the merger or from various epochs of nuclear activity. Deprojecting the surface brightness profile and using the best fit temperature and assuming $Z=0.5$, we find a hydrogen density of $\sim 10^{-3}$ $\mathrm{cm}^{-3}$. The surface brightness profile corresponding to this density model is overplotted onto Figure 8. The gas pressure in this sphere is $\sim 8.5 \times 10^{-13}$ dyne $\mathrm{cm}^{-2}$.

The discovery of a large scale gas halo is Cen A is entirely consistent with what we know about Cen A as the dominant member of a group. This halo has not been previously reported due to its angular extent (as much as a degree or more on the sky) and low surface brightness. Cen $\mathrm{A}$ is known to be the dominant member of a poor group with a total gravitating mass of $1-2 \times 10^{13} \mathrm{M}_{\odot}$ to a distance of $\sim 640 \mathrm{kpc}$ (Hesser et al. 1984; van den Bergh 2000). The total gas mass out to $35 \mathrm{kpc}$ (based on our density model above) is $\sim 3 \times 10^{9} \mathrm{M}_{\odot}$. There is likely to be considerable gas out to larger radii. Chandra observations of groups and clusters of galaxies show that baryon fraction is typically a few percent of the gravitating mass out to $r_{500}$ (Finoguenov et al. 2001; Khosroshabi et al. 2007; Sun et al. 2008). Therefore the total gas mass of Cen A is likely to be $2-3 \times 10^{13} \mathrm{M}_{\odot}$. Even out to $35 \mathrm{kpc}$, we are seeing only a small fraction of the Cen A IGM. A recent HI survey of Cen A dwarf ellipticals confirms the presence of the Cen A gas halo. Bouchard et al. (2007) found that many Cen A dwarf galaxies were devoid of $\mathrm{HI}$ and suggested that it had been ram pressure stripped by gas at density on the order of $10^{-3} \mathrm{~cm}^{-3}$. Such an extended halo around Cen A is entirely consistent with our X-ray observations.

\section{Interpretation}

Based on the spectra, we conclude that the X-ray filament consists of hot gas that lies along the southeastern boundary of the NML. Given the thermal spectra (for four of the five knots), we rule out a non-thermal (synchrotron or inverse-Compton scattering) origin for the X-ray knots. The proton density, pressure, mass, thermal energy, and sound crossing time for each knot (assuming the values from the best-fit collisional plasma model) are tabulated in Table 2. We have assumed spherical symmetry and unity filling factor for each of the features, and chosen a radius that roughly corresponds to the largest dimension for each feature. We also have assumed that $n_{e}=1.2 n_{H}$, appropriate for a plasma with sub-Solar elemental abundances. The lifetime is the sound crossing time of the knot and is defined as 
the radius divided by the sound speed (for $\gamma=5 / 3$ ). A smaller filling factor (i.e. if the gas is clumped and/or if considerable unresolved structure exists in a given feature) would increase the gas density and pressure of the individual clumplets, but decrease the total mass, thermal energy, and lifetime of the gas. The low elemental abundances found in the spectral fits are probably not realistic and likely the result of unresolved multi-temperature gas. In such cases, the best fit temperature usually represents a reasonable average temperature, but the low abundance would imply an artificially large gas density and pressure. We have scaled all the XSPEC normalizations, $N$, from the spectral fits to $Z=0.5$ Solar assuming that $N \propto Z$ to provide more realistic estimates of gas pressures, densities, and total masses.

The X-ray knots/bridge and associated optical emission features of the Cen A NML are analogous to similar features seen in the Extended Emission Line Region (EELR) of a large number of low redshift radio galaxies as well as many more powerful, distant radio galaxies (Baum et al. 1988). Individual examples of jet-cloud interactions studies with Chandra include the nearby 3C 277.3 (Tadhunter et al. 2000) and PKS 2153-699 (Lv et al. 2005; Young et al. 2005) as well as the more distant ( $\mathrm{z}=0.81$ ) 3C 265 (Solórzano-Iñarrea \& Tadhunter 2003). The EELR region is typically defined as the region $>1 \mathrm{kpc}$ and $<20 \mathrm{kpc}$ from the nuclei of radio galaxies so that it lies beyond the narrow line region (NLR) of the active nucleus but still within the host galaxy. In fact, the multi-phase gas features and regions of star formation in Cen A lie at somewhat greater distances from the nucleus (up to 30 $\mathrm{kpc}$ ) than the EELR region, nominally outside of the galaxy. Energy deposition into the ISM via shock-heating by radio outflows and photoionization from nuclear emission plays a key role in the overall energy budget of the galaxy and its evolution. Jet-induced star formation is also a common phenomenon seen in both nearby objects, including Cen A (Oosterloo \& Morganti 2005; van Breugel et al. 2004), and high redshift elliptical galaxies in the process of formation and undergoing their last epoch of significant star formation (Dopita et al. 2007). Such interactions may also be important in the regular refueling of nuclear activity (Tadhunter, Fosbury, \& Quinn 1989). A general review of this phenomenon is given in Tadhunter (2002).

There are a priori at least five plausible scenarios for the origin of the thermal gas filament of the Cen A NML: supernovae-driven winds from regions of jet induced star formation, photo-ionization from the beamed X-ray flux from the parsec scale jet, entrainment of hot ISM from the central region of the galaxy by the NML as it rises buoyantly, shock-heating of ambient gas due to the supersonic inflation of the NML, shock-heating of the ambient gas due to supersonic gas motions of the ISM, and warm/cold gas that is being directly heated by the jet. We discuss each of these possibilities in more detail below and find serious problems with each of the first five scenarios. The most likely possibility is that the X-ray emission originates from warm/cold gas that is shock heated by the jet. 
One important constraint on any physical model for the origin of the X-ray filament is the age of the NML. As elucidated below, there are two general possibilities: either NML is part of the current epoch of nuclear activity and has the same age as the inner radio lobes (roughly 3 Myrs - see Kraft et al. (2003) and Croston et al. (2009)), or the NML is part of a previous epoch of of nuclear activity unrelated to the inner radio features and has risen buoyantly to its present position (age is roughly 100-200 Myrs (Saxton, Sutherland, \& Bicknell 2003)). We favor a scenario in which the NML was formed as part of a previous epoch of nuclear activity that has been re-energized by the present outburst.

\subsection{Star Formation - Supernova-Driven Hot Gas Bubbles}

The large thermal energy and mass of the gas argues against the possibility that the X-ray filament and knots of the NML are the result of a supernovae-driven wind from a recent epoch of jet induced star formation. The total thermal energy of the filament is $\sim 10^{55-56}$ ergs, requiring $10^{4-5}$ supernovae over the last several million years to account for the hot gas. This is simply not plausible. There is a series of OB associations and recent star formation at the base of the NML in the vicinity of optical filament B, roughly $3.5^{\prime}(3.77$ kpc in projection) from knot N4 (Graham 1998; Mould et al. 2000). They estimate that roughly $1.2 \times 10^{4} \mathrm{M}_{\odot}$ of stars have formed in $\sim 16$ Myrs. This level of star formation is well below what would be required to create supernovae driven bubbles on the scale seen in the Cen A NML. There is no evidence for star formation on such a massive scale as required to produce SN-driven winds on the observed scale in the NML.

Contours from the X-ray image in Figure 4 have been overlaid onto an archival GALEX NUV image of Cen A in Figure9, The position of the OB associations created by compression of the HI cloud by the radio jet are clearly visible in the NUV GALEX image (Neff et al. 2003). There are no other significant associations of hot stars associated with any of the X-ray knots of the NML. There does not appear to be any region of enhanced star formation associated with the X-ray emitting gas. We further processed the GALEX image using a wavelet decomposition to remove all of the small-scale (i.e. point source) structure to search for diffuse emission. Again, there does not appear to be any significant enhancement of NUV emitting gas associated with the X-ray knots of the NML as there is for the star forming region at the base of the NML. Additionally, we examined archival Spitzer/MIPS observations of the NML region of Cen A for any evidence of star formation or stellar clusters that may be related to the X-ray emission and found none. We therefore reject the possibility that this gas is the result of a supernovae-driven wind. 


\subsection{Photoionization}

It is possible that the X-ray filament was created by photo-ionization from the beamed X-ray flux from the parsec scale jet at the nucleus. However, the observed X-ray flux (i.e. into our line of sight) from the accretion disk (Evans et al. 2004) is orders of magnitude too small to create X-ray knots $15-20 \mathrm{kpc}$ away. We therefore require that there be a substantially larger flux of beamed EUV/X-ray emission in the direction of the X-ray knots. The spectral resolution of Si detectors is not sufficient to distinguish between photoionized gas and collisionally excited gas at X-ray temperatures. We estimate the required luminosity using arguments commonly invoked to explain the presence of X-ray emitting gas in the vicinity of Seyfert nuclei. If the gas is optically thin to soft X-rays (certainly true in our case), the ionization structure is completely determined by the ionization parameter, $\xi$ (units of ergs $\mathrm{cm} \mathrm{s}^{-1}$ ), and is given by

$$
\xi=\frac{L_{n u c l}}{n_{H} d_{s}^{2}}
$$

where $L_{\text {nucl }}$ is the luminosity of the source of ionizing photons ( $\operatorname{ergs~s}^{-1}$ ), $n_{H}$ is the hydrogen density $\left(\mathrm{cm}^{-3}\right)$, and $d_{s}$ is the distance $(\mathrm{cm})$ from the source to the X-ray emitting cloud. The $\mathrm{X}$-ray spectrum of each of the knots is dominated by emission from the Fe $\mathrm{L}$ complex between 0.7 and $1.1 \mathrm{keV}$. For the photoionization spectrum to be dominated by this emission, $\xi \sim 100$ (Kallman \& McCray 1982; Kallman 1991; Weaver et al. 1995). The X-ray luminosity of the knot, $L_{k n o t}$, is given by

$$
L_{k n o t}=\frac{4 \pi}{3} n_{H}^{2} r^{3} \Lambda(\xi),
$$

where $r_{k n o t}$ is the radius of the knot and $\Lambda$ is the emissivity $\left(\operatorname{ergs~} \mathrm{cm}^{3} \mathrm{~s}^{-1}\right)$ of the photoionized gas. For $\xi \sim 100, \Lambda \sim 10^{-24}$ ergs $\mathrm{cm}^{3} \mathrm{~s}^{-1}$ (Kallman \& McCray 1982), and we find $n_{H} \sim$ $4.2 \times 10^{-2} \mathrm{~cm}^{3}$ (not surprisingly similar to the collisional case).

Using the observed values of X-ray flux for the brightest knot N1 $\left(F_{x}=5.6 \times 10^{-13}\right.$ ergs $\mathrm{cm}^{-2} \mathrm{~s}^{-1}$ in the 0.1-10.0 keV bandpass (unabsorbed), $L_{x}=9.1 \times 10^{38} \mathrm{ergs} \mathrm{s}^{-1}$ ) and at $\sim 30 \mathrm{kpc}$ from the nucleus the most distant, we require a nuclear luminosity of $\sim 6 \times 10^{46} \mathrm{ergs} \mathrm{s}^{-1}$, five orders of magnitude larger than the observed nuclear flux (Evans et al. 2004). In the unified scheme, Cen A is a misdirected BL Lac object (Morganti et al. 1991), so that the X-ray flux within a few degrees of the direction of the jet could be orders of magnitude larger than that we observe from the nucleus. This could be mitigated somewhat if the central source of photons is strongly beamed. In fact, (Morganti et al. 1991) claimed that beamed flux from the nucleus could explain the optical emission line filaments if the nuclear flux is $\sim 200$ times larger than the observed flux. Large boosts can be obtained on-axis for moderate values of $\Gamma$, but the five bright knots subtend an angle of $\sim 15^{\circ}$ projected on the sky and are misaligned relative to the pc-scale jet and the inner jet. Assuming that the X-ray filament occupies a 
cone with an opening half-angle of $\sim 7^{\circ}$, the required value of $\Gamma$ is $\sim 8$. This value of $\Gamma$ would boost the flux from the nucleus toward the NML by a factor of roughly 250, thus dropping the beam power (on one side) to $\sim 2.4 \times 10^{44} \mathrm{ergs} \mathrm{s}^{-1}$.

There are at least five serious problems for this model. First, this beamed flux would be easily observable due to electron scattering from the hot gas in the central few kpc of the galaxy. The optical depth to electron scattering, $\tau_{e s}$, is given by $\tau_{e s}=1.2 n_{H} \ell \sigma_{e s} \sim 10^{-5}$. The X-ray luminosity of the electron scattered photons would be $\sim 5 \times 10^{39} \mathrm{ergs} \mathrm{s}^{-1}$ for the gas in the central $5 \mathrm{kpc}$ of Cen A. This would be easily observable (the integrated X-ray luminosity of all of the hot gas in Cen $\mathrm{A}$ is only $\sim 10^{40} \operatorname{ergs~s}^{-1}$ ). Second, we have chosen the smallest possible solid angle for the beamed flux. The knots are probably spread over a larger solid angle in three dimensions, and the pc-scale jet is misaligned relative to the NML. The luminosity quoted above is then only a lower limit. Third, the luminosity and velocity of the jet are both uncomfortably large. We minimally require a beamed flux that is at least several times larger than that postulated by Morganti et al. (1992) to explain the inner optical filaments. Fourth, there is no evidence for any interaction between the beamed flux from the nucleus and cold/warm gas in the central regions of the galaxy, nor from any beamed flux on the counter-jet side. Such a large flux distributed over a fairly large solid angle should leave some observable signature on the cold/warm gas (e.g. other photoionized clouds or perhaps X-ray reflection nebulae similar to Sgr A*) in the central few kpc. Fifth, if the X-ray filament of the NML were the result of photoionization, it would make the close spatial relationship (in projection) to the radio lobe purely coincidental. The photoionization model is implausible in our view.

\subsection{Supersonic motions of the external ISM}

As a third scenario, we consider the possibility that the X-ray filament is created by the supersonic motion of the ambient ISM. As described in section 3.4, there is an extensive gas halo around Cen A extending at least to the position of the NML. Supersonic galaxy and cluster mergers have been well studied by Chandra (the Bullet cluster being the best example). Cen $\mathrm{A}$ is known to have undergone a fairly recent merger (several hundred million years ago - see the Introduction). In this model, the X-ray filament represents the shock front as the ISM moves supersonically to the NW. The NML could then have been re-energized by the shock. The shock is not currently visible in the NML as it would propagate rapidly through the lobe since the lobe's sounds speed is high relative to that of the ISM. There are several serious problems with this model. First, equating the ram pressure of the ambient gas $\left(1 / 2 \rho_{I S M} v_{I S M}^{2}\right)$ with the pressure of the knots $\left(p_{k n o t}\right.$ from Table 2$)$ to estimate the gas 
velocity $\left(v_{I S M}\right)$, we find that the gas must be moving at $\sim 1700 \mathrm{~km} \mathrm{~s}^{-1}$. This is roughly Mach 6 for the $k_{B} T=0.3 \mathrm{keV}$ ISM gas, implausibly large. Second, even if the merger took place at these velocities, it is unlikely that the velocity remains supersonic several hundred years after the merger. Additionally, there is no way that two small galaxies would be bound to each other if they approached each other with this velocity (i.e. the small spiral would have shot past Cen A, not fallen into the center). Fourth, if the NML has been shock heated, it would have to be in roughly pressure equilibrium with the filament, which does not appear to be the case. This scenario is highly implausible.

\subsection{Interaction with the Radio Lobe}

We next consider if the X-ray knots are produced by a direct interaction between the radio plasma of the NML and gas in the vicinity of or interior to the NML. We consider three scenarios including shock heating of the ambient ISM by the supersonic inflation of the lobe, entrainment by a buoyant bubble, and a direct interaction between the momentum of an unseen jet with cold clouds embedded in the NML. Before discussing each of these scenarios in detail, we first present a re-analysis and discussion of the existing radio data on the NML to constrain the relationship between the radio plasma and the hot gas. We computed the equipartition pressure of the NML using a C-band radio map. We assume that the NML is a cylinder of radius $6.5^{\prime}$ and length of $21.7^{\prime}$. The flux density of the NML is $25.5 \mathrm{Jy}$ at $5 \mathrm{GHz}$. Using standard assumptions about the low energy particle distribution (i.e. no protons and $\gamma_{\min }=100$ ), we find an equipartition pressure of $1.2 \times 10^{-12} \mathrm{dyn}_{\mathrm{cm}}^{-2}$ and an equipartition magnetic field, $B_{e q}$, of $\sim 7 \mu \mathrm{G}$.

Radio polarization maps could, in principle, constrain the relationship of the radio emitting plasma to the X-ray emitting gas via measurement of the rotation measure. The rotation measure map of the Cen A NML (Figure 5 of Morganti et al. (1999)) shows systematic variations of the rotation measure between $+40 \mathrm{rad} \mathrm{m}^{-2}$ and $-160 \mathrm{rad} \mathrm{m}^{-2}$. There are three arcminute-scale regions along the southeastern boundary of the lobe where the rotation measure changes from $-40 \mathrm{rad} \mathrm{m}^{-2}$ to $-160 \mathrm{rad} \mathrm{m}^{-2}$ on scales of tens of arcseconds. None of these

regions coincide with an X-ray knot, so unfortunately no information about the position of the knots relative to the radio plasma can be drawn. Additionally, the implied minimum magnetic field (assuming the kpc-scale features of the X-ray knots associated with the NML and densities taken from Table 2) required to create the observed rotation measure is on the order of $10 \mu \mathrm{G}$. This magnetic field is not unrealistic, although somewhat larger than the few $\mu \mathrm{G}$ fields one might naively expect. The pressure of the knots would still be dominated by the hot gas. It is in our view more likely that unrelated gas on much larger spatial scales 
(with correspondingly lower magnetic field and lower density) is responsible for the observed variations in rotation measure. Morganti et al. (1999) point out that the X-ray filament is associated with the region of lowest fractional polarization, suggesting that the hot gas is either a screen in front of the lobe or intermixed with the lobe plasma.

\subsubsection{Supersonic inflation of the $N M L$}

In the scenario where that the X-ray filament has been created by the supersonic inflation of the NML, the NML is still being powered by the active nucleus and cold or warm gas has been shock-heated to keV temperatures. The NML is then an energy-driven bubble driving a shock into the ambient gas. Such a model has been invoked to explain the hot shell of gas around the SW inner lobe of Cen A (Kraft et al. 2003), a large number of shocks seen in galaxy groups and clusters of galaxies (Forman et al. 2005; Nulsen et al. 2004; McNamara et al. 2005), and hard X-ray emission associated with CSS radio source 3C 303.1 (O'Dea et al. 2006). In this case, the knots/bridge of the NML were originally warm/cold gas in rough hydrostatic equilibrium with the hot ISM that was shock heated by the NML. There are several attractive features of this scenario. First, the sound crossing time of the knots is roughly consistent with the age of the shock around the SW radio lobe (roughly 3 Myrs, see Kraft et al. (2003); Croston et al. (2009)). If this age also represents the approximate age of the NML, the NML and the SW (inner) lobe are part of the same outburst. The momentum imparted to dense clouds in a stratified atmosphere by the passage of a strong shock is small, and the sites of the knots could represent the positions of dense clouds that have been shock heated. The tenuous filament between the knots then represents material that has been ablated from the dense clouds.

The main problem with this scenario is that the equipartition pressure of the radio lobe is more than an order of magnitude below that of the knots. This is, of itself, not a serious problem. In fact, we have shown that the SW inner lobe must be far from equipartition and greatly overpressurized relative to the ambient medium (Kraft et al. 2003; Croston et al. 2009). In addition, Chandra and XMM-Newton observations of the hot gas atmospheres surrounding radio lobes show that the equipartition pressure of lobes can be more than an order of magnitude less than that of the ambient pressure, particularly for FR I sources. It is difficult to see how the pressure of the NML can be substantially less than that of shock-heated features which the lobe has already overrun (i.e. they are internal to the lobe). Additionally, at the equipartition pressure, the NML is currently in rough pressure equilibrium with the ambient ISM (based on an extrapolation of the surface brightness

profile of the ISM derived from the Chandra image to the distance of the NML). Increasing 
the equipartition energy and pressure of the lobe to roughly match that of the X-ray knots would then imply the NML is enormously overpressurized relative to the ambient ISM. Additionally, the total energy of the NML, at the equipartition value, is roughly the same as that of the SW (inner) lobe. If the NML were in rough pressure equilibrium with the knots, the internal energy of the NML would be more than an order of magnitude larger than the SW inner lobe. Thus the knots and the SW lobe could not be from the same outburst, but the NML could also not be the result of an earlier epoch of nuclear activity given its short lifetime. This scenario leads to a contradiction. Additionally, the lack of any counterpart to the NML on the southwestern side of Cen A becomes even more puzzling.

\subsubsection{Entrainment}

A fourth scenario is that the X-ray filament consists of material that is entrained by the NML as it is driven or rises buoyantly in the hot gas atmosphere (Saxton, Sutherland, \& Bicknell 2003). The entropy of the gas in the knots is roughly consistent with that in the center of Cen A, and we typically expect only adiabatic compression if the gas is entrained via Kelvin-Helmholtz instabilities in the linear regime. There are several serious problems with this interpretation, however. Most importantly, the short lifetimes and tremendous overpressurization of the X-ray knots relative to the NML make the model of buoyant entrainment untenable. The equipartition magnetic field of the radio lobe is $\sim 7 \mu \mathrm{G}$, the equipartition pressure of the lobe is $\sim 1.2 \times 10^{-12} \mathrm{dyn}_{\mathrm{cm}^{-2}}$. This is more than an order of magnitude lower than the thermal pressures of the various knots. Dissipation and heating will invariably occur in gas that has been entrained via Kelvin-Helmholtz instabilities, but it is difficult to see how such large pressure gradients could be formed and stably maintained. The buoyant rise time of the NML from the nucleus is $\sim 170$ Myrs (Saxton, Sutherland, \& Bicknell 2003). If the X-ray knots are unconfined, they will expand at roughly their internal sound speed until they reach pressure equilibrium with the lobe (or other ambient gas that dominates the pressure). The sound crossing time of the X-ray knots is more than an order of magnitude smaller than the buoyant rise time to the position of the NML. The only way that this scenario is plausible is if the NML is far from equipartition.

\subsubsection{Cold gas directly impacted by the jet}

The remaining viable scenario to explain the X-ray filament is that the X-ray knots and bridge are (or were) directly embedded in the jet. That is, cold, dense clouds lie directly in the path of the jet and are in direct contact with the jet momentum. This is the only 
scenario that can reasonably reproduce the observed features of the X-ray filament without requiring unrealistically large input energies or inconsistencies related to timescales. The NML expands to the NW of the X-ray filament, so it is reasonable to speculate that the jet was deflected to the NW by the interaction with the cold gas (i.e. the collision that created the X-ray filament was oblique with the jet generally lying to the NW of the cold gas).

Simulations of jet-cloud interactions demonstrate that a dense obstacle can deflect a jet (De Young 1991; De Young 2002; Higgins et al. 1999; Wang et al. 2000). In fact, many features of these simulations qualitatively reproduce structures seen in the X-ray filament of the NML. Strong shocks will be driven into the dense clouds. The X-ray knots of the NML could represent denser clouds of gas that have been shock heated by an oblique interaction with the jet. The filamentary bridge structure between the knots is then gas that has been heated and ablated from the dense clouds. Simulations of the propagation of a supersonic jet through a stratified medium consisting of a hot, low density gas that surrounds cooler, denser clouds show the underlying flow patterns can be quite complex in the case in which the filling factor of the cold gas is large (Saxton et al. 2005). The momentum of the jet tends to propagate through a number of small channels as the dense clouds are shock-heated and subsequently ablated. Ablation and shock-heating of gas is entirely consistent with our data; each of the bright knots could be a cloud and the bridge connecting the knots could be ablated gas that has been swept along the direction of the flow. This scenario naturally explains the observed anti-correlation of the X-ray and radio knots of the NML. The radio plasma does not penetrate the clouds but is compressed into the channels between them with a significant increase in their radio brightness due to compression of the magnetic field.

This model requires that the NML is actively powered by the central engine, but whether it is part of the current outburst or a re-energized bubble from a previous outburst is not clear. As we describe below, we favor the latter scenario. It also requires that the NE inner lobe is really a 'jet' in the sense that it acts as a nearly lossless tunnel through which the collimated material from the nucleus flows out to the NML. The term 'NE inner lobe' is therefore somewhat of a misnomer, it is really a jet with at least two bends in it: the first at the transition between the inner jet and inner lobe (where the flow must become subsonic relative to its internal sound speed), and a second as the inner lobe bends into the large scale radio jet. Subsonic or transonic gas motions of the external medium are likely the cause of the jet bends (Kraft et al. 2008). We also note that we implicitly assume in the discussion that follows that the jet mechanical power is equal on either side of the AGN (both instantaneously and in a time averaged sense over a long period). If the jet power/momentum were unbalanced on the two sides of the AGN, the central SMBH would be kicked out of the central region of the galaxy on a short timescale. This is a conservative assumption as there are no known examples of this process occurring. 
The energetics of the NML and the SW inner lobe are surprisingly similar, strengthening the argument that they are both related to the current outburst. Assuming the geometry for the NML used in the equipartition argument above and the equipartition pressure, the enthalpy (for $\gamma=4 / 3$ ) of the NML is $\sim 3 \times 10^{56} \mathrm{ergs}$. This is really a lower limit to the total enthalpy, but as we argued above, the NML is probably not far from equipartition. The pressure of the SW inner lobe is $\sim 1.6 \times 10^{-11} \mathrm{dyn} \mathrm{cm}^{-2}$ (Kraft et al. 2003; Croston et al. 2009). Assuming a simple elliptical geometry for this lobe, its enthalpy is $\sim 6 \times 10^{56}$ ergs. Roughly half the energy that has gone into inflating the SW inner lobe is required to inflate the NML. Additionally, the total thermal energy of the five knots in the NML is $\sim 2.6 \times 10^{55}$ ergs. Thus only five percent of the energy required to inflate the NML must go into shockheating cold gas to create the X-ray knots. Based on energetics, it is entirely plausible that the NML and the SW inner lobe are part of the outburst, and only a small fraction of the outburst energy must be diverted to shock-heating the X-ray emitting knots.

The problem with associating the NML directly with the current outburst is that the average inflation speed of the bubble must have been considerably higher than the expansion velocity of the SW inner lobe given that the NML is both further away from the nucleus and considerably larger than the SW inner lobe. At minimum pressure the NML appears to be in rough pressure equilibrium with the ambient gas, and there is no evidence for a shell of hot gas around the NML that would be expected if it had recently driven a shock. Thus, it is more likely that the NML does indeed represent a previous epoch of nuclear activity. A direct channel between the NML and NE inner lobe has been opened and the NML is now being powered. This requires that there is an unseen radio bubble lying several tens of $\mathrm{kpc}$ to the SW of the active nucleus of Cen A that was created in this earlier epoch of nuclear activity. Non-detection of a radio bubble at hundreds of $\mathrm{MHz}$ and/or $\mathrm{GHz}$ frequencies does not conclusively rule out the existence of a radio bubble. Chandra observations of NGC 4636 show clear evidence of shocks being driven into the ISM by unseen radio bubbles/jets (Jones et al. 2002; Baldi et al. 2008). Ghost cavities have also been observed in a number of objects in which there are clear depressions in the Xray emission of group/cluster gas associated with the inflation of radio lobes but faint or undetected radio bubbles (McNamara et al. 2001; Choi et al. 2004). Radiative or adiabatic losses could easily account for the near invisibility of a putative southwestern counterpart to the NML. Interestingly a short $(14 \mathrm{ks})$ archival ROSAT PSPC observation of Cen A shows marginal evidence for a ghost cavity to the southwest of the nucleus. A smoothed, exposure corrected ROSAT PSPC image of Cen A in the 0.5-1.1 keV band is shown in Figure10. The white arrows delineate what may be a ghost cavity. If the NML is the result of a previous outburst, our conclusions are largely unchanged. The energy required to re-energize $\mathrm{GHz}$ emitting particles of the NML is obviously less than the total enthalpy of the lobe, requiring 
an even smaller fraction of the energy of the current outburst.

One obvious question that presents itself in the jet-cloud model is that of where the cold gas originated. We consider three possible scenarios. First, we consider a model in which a direct interaction between the jet and the HI cloud at the base of the NML imparted sufficient momentum to drive the knots out to their current position. Again we consider knot N1 as the most distant and therefore demanding case. The mass of this feature is $\sim 3.5 \times 10^{6} M_{\odot}$ and it lies roughly $10 \mathrm{kpc}$ from the $\mathrm{HI}$ cloud. Its lifetime is $\sim 3 \mathrm{Myrs}$, so that is must have a velocity of $\sim 3000 \mathrm{~km} \mathrm{~s}^{-1}$ to reach its position relative to the HI cloud. The acceleration required to achieve this velocity in this time is $\sim 3 \times 10^{-6} \mathrm{~cm} \mathrm{~s}^{-2}$. If we assume that the jet is relativistic (i.e. light), the mechanical energy required to accelerate knot N1 to $3000 \mathrm{~km}$ $\mathrm{s}^{-1}$ in 3 Myrs is $\sim 1.2 \times 10^{59}$ ergs or a mechanical power of $\sim 1.3 \times 10^{45} \mathrm{ergs} \mathrm{s}^{-1}$. This is two orders of magnitude larger than the mechanical power of the SW lobe, and is in any case more typical of FR II radio galaxies. The energy and momentum required to push cold gas from the HI cloud to knot N1 is implausibly large, and we conclude that most of the gas in the X-ray filament did not originate in this HI cloud. On the other hand, we could assume that the jet is heavily mass-loaded. It may have started as a relativistic plasma, but has swept up considerable mass (perhaps via Kelvin-Helmholtz instabilities along the jet/ISM interface or from stellar mass loss of stars embedded in the jet). The energy requirements are then greatly reduced, but the jet density required is comparable to or greater than the density of the ambient ISM and thus implausibly high. The X-ray knots must have been shock-heated in situ near to their present positions.

Second, the knots could have recently drifted into the jet. The edges of the clouds are then continuously torn off as they propagate into the beam. Given the age of the knots $\left(3 \times 10^{6} \mathrm{yrs}\right)$ and assuming they are rotating in the gravitational potential of the galaxy, they could only have travelled $\sim 1 \mathrm{kpc}$ in the lifetime of the jet (i.e just outside the jet). This scenario would naturally explain the bridge of emission between the knots as it represents material ablated by the jet. This model also seems unlikely as it requires a chain of knots originally located just outside the NML to drift in. A third scenario (and most likely in our view) would be that the clouds drifted into the NML prior to the current outburst and are now being lit up by the current epoch of re-energization of the NML. Transonic or subsonic gas motions of the external medium can easily push (and deform) the NML over a timescale of hundreds of megayears.

In this jet-cloud interaction scenario, the jet must survive two abrupt bends to provide a collimated outflow to the NML. Light, highly supersonic jets cannot be bent without typically disrupting the jet on a short timescale, demonstrating that the jet must be subsonic at the end of the inner jet. Hydrodynamic modeling of mass loading of the jet, assuming it is 
pressure confined by the external medium, shows that the jet likely decelerates to subsonic velocities just as it enters the NE inner lobe (P. Nulsen et al. in preparation). Once the jet becomes subsonic, external subsonic or transonic gas motions can easily deform the jet. Based on a deep Chandra observation, we have already shown the existence of a surface brightness discontinuity in the gas at the base of the NE lobe and speculated that the NE inner lobe may have inflated in a cross-wind (Kraft et al. 2008). Observationally, there are a large number of examples of jets remaining well collimated after a bend on scale of pcs (e.g. VLBI observations of BL Lac jets) to tens of kpc (e.g. Her A). The bending of jets in wide and narrow angle tailed galaxies observed in clusters of galaxies are believed to be the result of ram pressure bending the jets (Pinkney et al. 1994), although these jets typically do not remain well collimated. Once a stable connection between the NE inner lobe and the NML is formed, energy can be rapidly transferred to the NML as the velocity of the jet, while subsonic, is still likely to be considerably larger than any large scale dynamical motions external to the jet. The large scale jet in Cen A connecting the NE inner lobe to the NML shows no evidence of decollimation. In fact, the opening angle of the LSJ appears to be constant between the NE inner lobe and the NML, qualitatively consistent with the large scale uniform density gas halo.

Additionally, it is not easy to understand how the bridge of X-ray emission extends $5 \mathrm{kpc}$ in projection beyond knot N1 and beyond the radio contours of the NML. By the same argument above, transonic motions of the external medium can only push the NML $\sim 1 \mathrm{kpc}$ in $3 \times 10^{6} \mathrm{yrs}$. The actual boundaries of the NML to the northeast and northwest are not well defined in the radio maps. The NML opens into much larger scale radio emission extending hundreds of $\mathrm{kpc}$ from the nucleus without any clearly defined interface. We can only speculate that the extension of the X-ray filament beyond knot N1 and the observed radio contours of the NML implies that the jet extends beyond the visible radio emission bending east from knot N1 (i.e. the radio emission shown on Figures 1 and 6 from the NML does not entirely define the location of the jet).

Finally as a minimal consistency check, we require that the pressure of the individual knots be on the order of or less than the ram pressure of the jet, or $p_{k n o t}<2 P_{j e t} / v_{j} A$ where $p_{k n o t}$ is the pressure of a knot, $P_{j e t}$ is the jet power, $A$ is the cross-sectional area of the knot, and $v_{j}$ is the jet velocity. In particular, we require that the upper limit to the speed of the jet is much larger than the thermal speed of the clouds so that the timescale for the jet interaction is smaller than any dynamical timescale for a cloud. From Tables 1 and 2 , the pressure of a typical cloud is $\sim 2 \times 10^{-11} \mathrm{dyn} \mathrm{cm}^{-11}$ with radius $\sim 1 \mathrm{kpc}$. We estimate the power of the jet $\left(6 \times 10^{42} \mathrm{ergs} \mathrm{s}^{-1}\right)$ based on the enthalpy of the SW inner lobe and the velocity of the shock (Kraft et al. 2003; Croston et al. 2009). For these values of the knot pressure, knot cross-section, and jet power, we find that $v<0.7 c$. Even if only a fraction 
$(<10 \%)$ of the jet power is intercepted by the X-ray knots, the jet velocity can easily be thousands or tens of thousands of $\mathrm{km} \mathrm{s}^{-1}$, well above any thermal velocity of any of the knots.

\section{Summary and Conclusions}

We present results from a $40 \mathrm{ks} X M M$-Newton observation of an X-ray filament associated with the Cen A Northern Middle Radio Lobe. Our conclusions can be summarized as follows:

1. The filament is composed of at least five discrete knots of emission connected by a narrow thread that lies along the southeast boundary of the radio lobe.

2. The spectra of all the knots and the connecting thread are well described by thermal models with low abundance, thus confirming that the emission is from hot gas.

3. We also report the discovery of a large scale X-ray emitting corona around Cen A.

4. Our preferred model for the origin of this X-ray filament is a jet-cloud interaction. Cold, dense clouds have been shock heated to X-ray temperatures.

5. It is likely that the NML was created by a previous outburst, but it is currently being powered by the ongoing nuclear outburst if our preferred model for the X-ray filament is correct.

The offsets between the optical emission-line gas and the X-ray emitting gas in the Cen A NML have important implications for our understanding of both the EELR in other radio galaxies (see references above) and the interaction of the relatively weak jets of Seyfert galaxies with the the ambient multiphase gas of the host galaxy (Evans et al. 2006; Croston et al. 2008). In particular, the spatial relationship between the radio jet, the X-ray emitting gas, and the warm optical emission-line gas is often not clear in these more distant galaxies. In the Cen A NML, we find that the optical emission-line gas is not co-spatial and intermixed with the X-ray emitting gas. However the jet is interacting with the ambient gas to create the observed filaments, the warm gas is clearly separated from the X-ray emitting gas even though both are greatly overpressurized relative ambient coronal gas and therefore short-lived.

There are at least four further observational programs that could be undertaken to confirm the results and interpretations presented here. First, a deep study of the large-scale 
X-ray corona would confirm or reject the presence of the ghost cavity hinted at in the ROSAT observation. A detailed study of the morphology and thermodynamic parameters of the gas in the vicinity of this cavity could constrain its age and total energy and would provide an important comparison of the results presented here. Second, there may well be considerably more HI around Cen A than that detected by previous studies. HI observations of early-type galaxies around the periphery of the Virgo cluster show little evidence for HI, but the ambient gas density is high enough that ram pressure stripping may be important in removing HI from galaxy halos. Cen A is in an isolated environment. so primordial HI may well survive into the present epoch. Schiminovich et al. (1994) find $1.5 \times 10^{8} M_{\odot}$ of HI in a shell out to $15^{\prime}$ from the nucleus. The X-ray filament lies beyond this shell, but the filament mass is small compared to the known HI. Scaling by the solid angle subtended by the NML, there could easily be an additional $\sim 10^{8} M_{\odot}$ of HI more than $15^{\prime}$ from the nucleus. The existing HI survey of the Cen A group (Banks et al. 1999) is not sensitive to the relatively small amounts of cold gas that are in the NML. A deeper, wider HI survey is required to evaluate the possible existence of this cold gas. Fourth, a more sensitive, higher resolution radio polarization study combined with the XMM-Newton data presented here, could conclusively determine the spatial relationship of the hot gas and the radio plasma of the NML. Finally, deep Chandra observations of the individual knots will give unique insights into the dynamics of the jet-cloud interaction. The unphysically low values found for the elemental abundances in the spectral fits suggest the presence of unresolved temperature structure in each of the knots. This structure would only be resolved with Chandra (on-axis), facilitating a unique comparison with hydrodynamic simulations of jet-cloud interactions.

Finally we note that this phenomenon would be nearly unobservable in most other radio galaxies given the relatively low luminosity of the X-ray knots. It is possible that the jetcloud interaction is a relatively common occurrence, and that we haven't observed many such examples simply due to a lack of sensitivity. If Cen A were 20 times futher away (70 Mpc), only a few tens of counts would be detected from the knots and none of the substructure would be observable. Because of the distance of the X-ray filament from the nucleus (15$30 \mathrm{kpc}$ ) and the inability to distinguish thermal from non-thermal models with such few counts, it is likely that the X-ray emission would be attributed to the synchrotron process. Additionally, Cen $\mathrm{A}$ is a relatively low power radio galaxy compared with many of the more distant $3 \mathrm{C}$ radio galaxies. In the more powerful radio galaxies, the jets are likely to remain light and fast to fairly large distances. In Cen A, the fact that the jet apparently bends (twice) between the inner jet and the NML suggests that the jet is transonic or subsonic and may therefore have swept up considerable mass. The jet impacting the clouds in the NML may therefore be both slower and denser than the jets of more powerful radio galaxies. Any jet/cloud interaction in the latter category of sources may simply result in the rapid 
destruction and incorporation of the cloud into the jet as it is shock-heated to temperatures well above the soft X-ray band.

\section{Acknowledgments}

This work was supported by NASA contract NAS8-03060, the Chandra X-ray Cen-

ter, and the Smithsonian Astrophysical Observatory. MJH thanks the Royal Society for a research fellowship. 


\section{REFERENCES}

Baldi, A. et al. 2008, ApJ, submitted.

Banks, G. D. et al. (1999), ApJ, 524, 612.

Baum, S. A., Heckman, T., Bridle, A., van Breugel, W., Miley, G. 1988, ApJS, 68, 643.

Blanco, V. M., Graham, J. A., Lasker, B. M., Osmer,, P. S. 1975, ApJ, 198, L63.

Bouchard, A., Jerjen, H., Da Costa, G. S., \& Ott, J. 2007, ApJ, 133, 261.

Burns, J. O., Feigelson, E. D., \& Schreier, E. J. 1983, ApJ, 273, 128.

Choi, Y. Y., Reynolds, C., Heinz, S., Rosenberg, J. L., Perlman, E. S., \& Yang, J. 2004, ApJ, 606, 185.

Croston, J. H., Hardcastle, M. J., Harris, D. E., Belsole, E., Birkinshaw, M., \& Worrall, D. M. 2005, ApJ, 626, 733.

Croston, J. H., Hardcastle, M. J., Kharb, P., Kraft, R. P., \& Hota, A. 2008, ApJ, 688, 109.

Croston, J. H., et al. 2009, MNRAS, astroph-0901.1346.

Dickey, J. M., \& Lockman, F. J. 1990, ARA\&A, 28, 215.

De Young, D. S. 1991, ApJ, 371, 69.

De Young, D. S. 2002, The Physics of Extragalactic Radio Sources (Chicago: University of Chicago Press).

Dopita, M. et al. (2007), Ap\&SS, 311, 305.

Evans, D. A., Kraft, R. P., Worrall, D. M., Hardcastle, M. J., Jones, C., Forman, W. R., \& Murray, S. S. 2004, ApJ, 612, 786.

Evans, D. A., Lee, J. C., Kamenetska, M., Gallagher, S. C., Kraft, R. P., Hardcastle, M. J., Weaver, K. A. 2006, ApJ, 653, 1121.

Evans, I., \& Koratkar, A. 2004, ApJ, 617, 209.

Feigelson, E. D., Schreier, E. J., Delvaille, J. P., Giacconi, R., Grindlay, J. E., \& Lightman, A. P. 1981, ApJ, 251, 31.

Ferrarese, L., Mould, J. R., Stetson, P. B., Tonry, J. L., Blakeslee, J. P., \& Ajhar, E. A. 2007, ApJ, 654, 186. 
Finoguenov, A., Reiprich, T. H., \& Böhringer, H. 2001, A\&A, 368, 749.

Forman, W. R. et al. (2005), ApJ, 635, 894.

Graham, John A. 1998, ApJ, 502, 245.

Hardcastle, M. J., Worrall, D. M., Kraft, R. P., Forman, W. R., Jones, C., \& Murray, S. S. 2003, ApJ, 593, 169.

Hardcastle, M. J. et al. 2007, ApJ, 670, 81.

Hesser, J. E., Harris, H. C., van den Bergh, S., \& Harris, G. L. H. 1984, ApJ, 276, 491.

Higgins, S. W., O’Brien, T. J. \& Dunlop, J. S. 1999, MNRAS, 309, 273.

Israel, F. P. 1998, Astron. \& Astrophys. Rev., 8, 237.

Jones, C., Forman, W., Vikhlinin, A., Markevitch, M., David, L., Warmflash, A., Murray, S., \& Nulsen, P. E. J. 2002, ApJ, 567, 115.

Junkes, N., Haynes, R. F., Harnett, J. I., \& Jauncey, D. L. 1993, A\&A, 269, 29.

Kallman, T. R. \& McCray, R. 1982, ApJS, 50, 263.

Kallman, T. R. 1991, Iron Line Diagnostics in X-ray Sources, 385, 87.

Khosroshabi, H. G., Ponman, T. J., \& Jones, L. R. 2007, MNRAS, 377, 595.

Kraft, R. P., Forman, W. R., Jones, C., Murray, S. S., Hardcastle, M. J., \& Worrall, D. M. 2002, ApJ, 569, 54.

Kraft, R. P., Vzquez, S. E., Forman, W. R., Jones, C., Murray, S. S., Hardcastle, M. J., Worrall, D. M., \& Churazov, E. 2003, ApJ, 592, 129.

Kraft, R. P., Nulsen, P. E. J., Birkinshaw, M., Worrall, D. M., Penna, R. F., Forman, W. R., Hardcastle, M. J., Jones, C., \& Murray, S. S. 2007, ApJ, 665, 1129.

Kraft, R. P. et al. 2008, ApJ, 677, L97.

Ly, C., De Young, D. S., \& Bechtold, J. 2005, ApJ, 618, 609.

Malin, D., Quinn, P. J, \& Graham, J. A. 1983, ApJ, 272, L5.

McNamara, B. R., et al. 2001, ApJ, 562, L149. 
McNamara, B. R., Nulsen, P. E. J., Wise, M. W., Rafferty, D. A., Carilli, C., Sarazin, C. L., \& Blanton, E. L. 2005, Nature, 433, 45.

Morganti, R., Robinson, A., Fosbury, R. A. E., di Serego Alighieri, S., Tadhunter, C. N., \& Malin, D. F. 1991, MNRAS, 249, 91.

Morganti, R., Fosbury, R. A. E., Hook, R. N., Robinson, A., and Tsvetanov, Z. 1992, MNRAS, 256, 1r.

Morganti, R., Killeen, N. E. B., Ekers, R. D., \& Oosterloo, T. A. 1999, MNRAS, 307, 750.

Morini, M., Anselmo, F., and Molteni, D. 1989, ApJ, 347, 750.

Mould, J. R., et al. 2000, ApJ, 536, 266.

Neff, S. G., Schiminovich, D., Martin, C. D., \& GALEX Science Team 2003, BAAS, 35, 1364.

Nulsen, P. E. J., Hambrick, D. C., McNamara, B. R., Rafferty, D., Birzan, L., Wise, M. W., \& David, L. P. 2004, ApJ, 625, L9.

O’Dea, C. P., Mu, B., Worrall, D. M., Kastner, J., Baum, S., de Vries, W. H. 2006, ApJ, 653, 1115.

Oosterloo, T. A., \& Morganti, R. 2005, A\&A, 429, 469.

Pinkney, J., Burns, J. O., \& Hill, J. M. 1994, AJ, 108, 2031.

Quillen, A. C., de Zeeuw, P. T., Phinney, E. S., \& Phillips, T. G. 1992, ApJ, 391, 121.

Saxton, C.J., Sutherland, R. S., \& Bicknell, G. V. 2003, ApJ, 563, 103.

Saxton, C. J., Bicknell, G. V., Sutherland, R. S., and Midgley, S. 2005, MNRAS, 359, 781.

Schiminovich, D. van Gorkom, J. H., van der Hulst, J. M., \& Kasow, S. 1994, ApJ, 423, L101.

Solórzano-Iñarrea, C. \& Tadhunter, C. N. 2003, MNRAS, 340, 705.

Soria, R. et al. 1996, ApJ, 465, 79.

Sun, M., Voit, G. M., Donahue, M., Jones, C., Forman, W., \& Vikhlinin, A. astro-ph 0805.2320.

Tadhunter, C. N., Fosbury, R. A. E., \& Quinn, P. J. 1989, MNRAS, 240, 225. 
Tadhunter, C. N., Villar-Martin, M., Morganti, R., Bland-Hawthorn, J. \& Axon, D. 2000, MNRAS, 314, 849.

Tadhunter, C. N. 2002, Revista Mexicana de Astronomia y Astrofisica Conference Series, 13, 213.

van den Bergh, S. 2000, AJ, 119, 609.

van Breugel, W., Fragile, C., Croft, S., de Vries, W., Anninos, P., \& Murray, S. 2004, The Interplay Among Black Holes, Stars and ISM in Galactic Nuclei, 222, 485.

Wang, Z., Wiita, P. J., \& Hood, J. S. 2000, ApJ, 534201.

Weaver, K. A., Mushotzky, R. F., Serlemitsos, P. J., Wilson, A. S., Elvis, M., \& Briel, U. 1995, ApJ, 442, 597.

Young, A. J., Wilson, A. S., Tingay, S. J., \& Heinz, S. 2005, ApJ, 622, 830. 


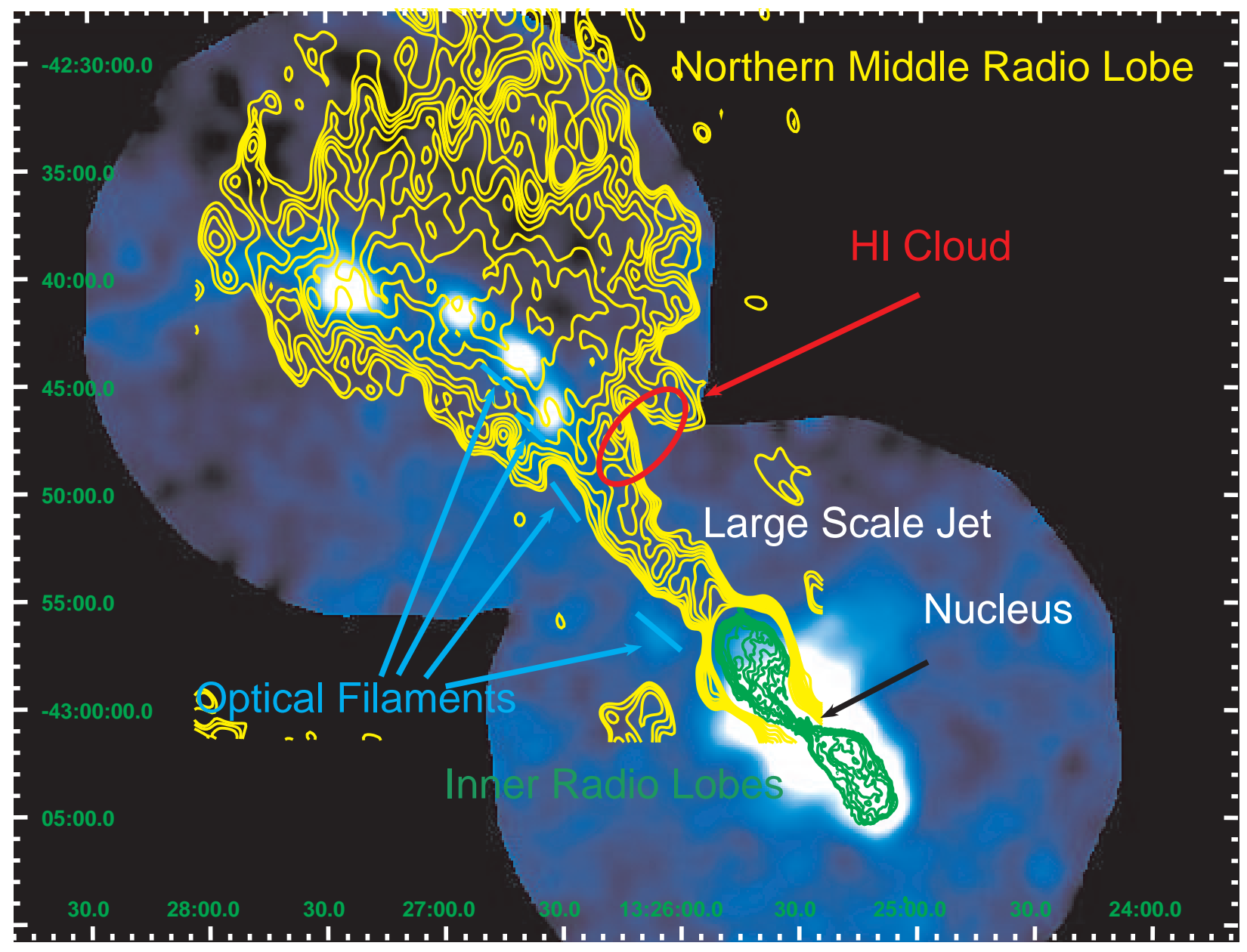

Fig. 1.- Exposure corrected, Gaussian smoothed XMM-Newton image of the inner jet/lobe and NML regions of Cen $\mathrm{A}$ in the $0.3-1.0 \mathrm{keV}$ band pass. The inner jet and inner radio lobes are shown with the green contours (Burns, Feigelson, \& Schreier 1983), the NML as the yellow contours (Morganti et al. 1999). The red circle denotes the approximate position of the HI cloud located at the base of the NML (Schiminovich et al. 1994; Oosterloo \& Morganti 2005). The position of the active nucleus is shown with the black arrow. The approximate positions of the inner and outer optical filaments (Blanco et al. 1975 ; Morganti et al. 1999) are shown as the cyan lines. 


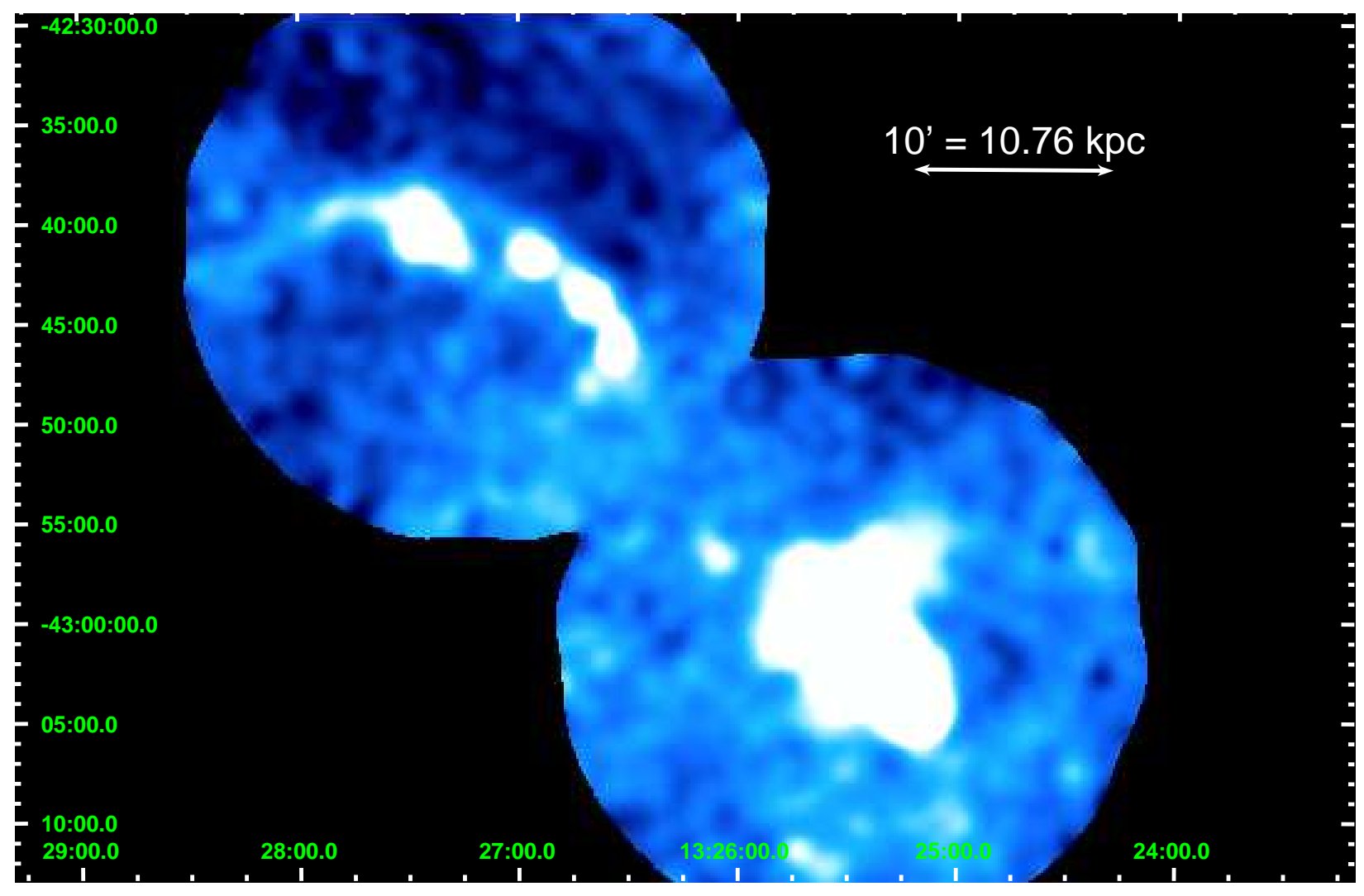

Fig. 2.- Exposure corrected, Gaussian smoothed XMM-Newton image of the inner jet/lobe and NML regions of Cen $\mathrm{A}$ in the $0.3-1.0 \mathrm{keV}$ band pass. This is the same image as shown in the previous figure without the radio contours and a slightly different color stretch to enhance the appearance of the large scale diffuse emission. Notice the surface brightness contrast to the NW and SE of the X-ray filament. The light blue and black (the level of the sky background) colors correspond to surface brightness of approximately 3.2 and $5.2 \times 10^{7}$ $\operatorname{cts~s}^{-1} \operatorname{arcsec}^{-2}$. 


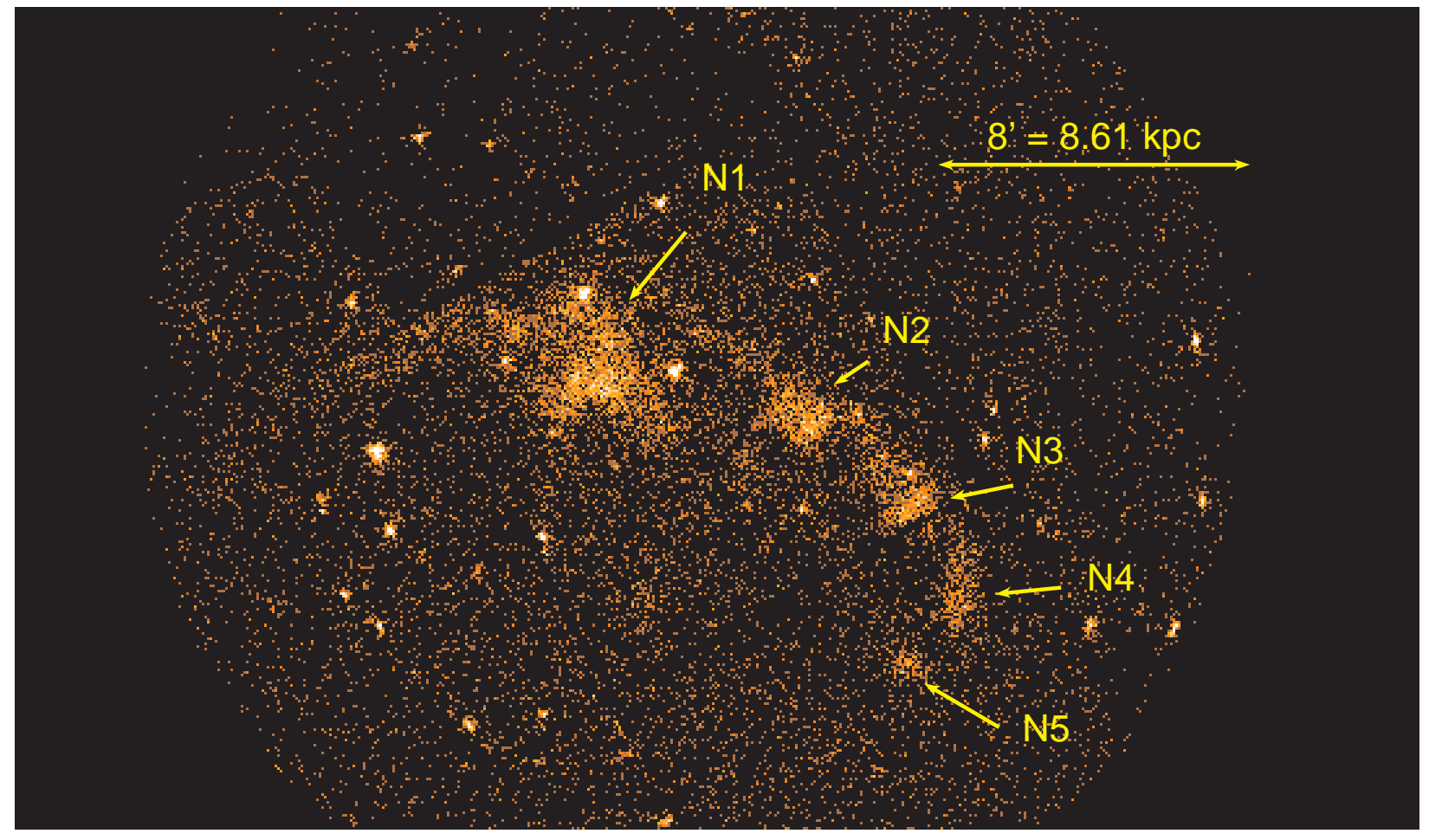

Fig. 3.- Unsmoothed XMM-Newton image of the Cen A NML (MOS1+MOS2 co-added) in the 0.5-2.0 keV band. The five X-ray knots are labeled N1 through N5. All five are spatially extended at the resolution of XMM-Newton. 


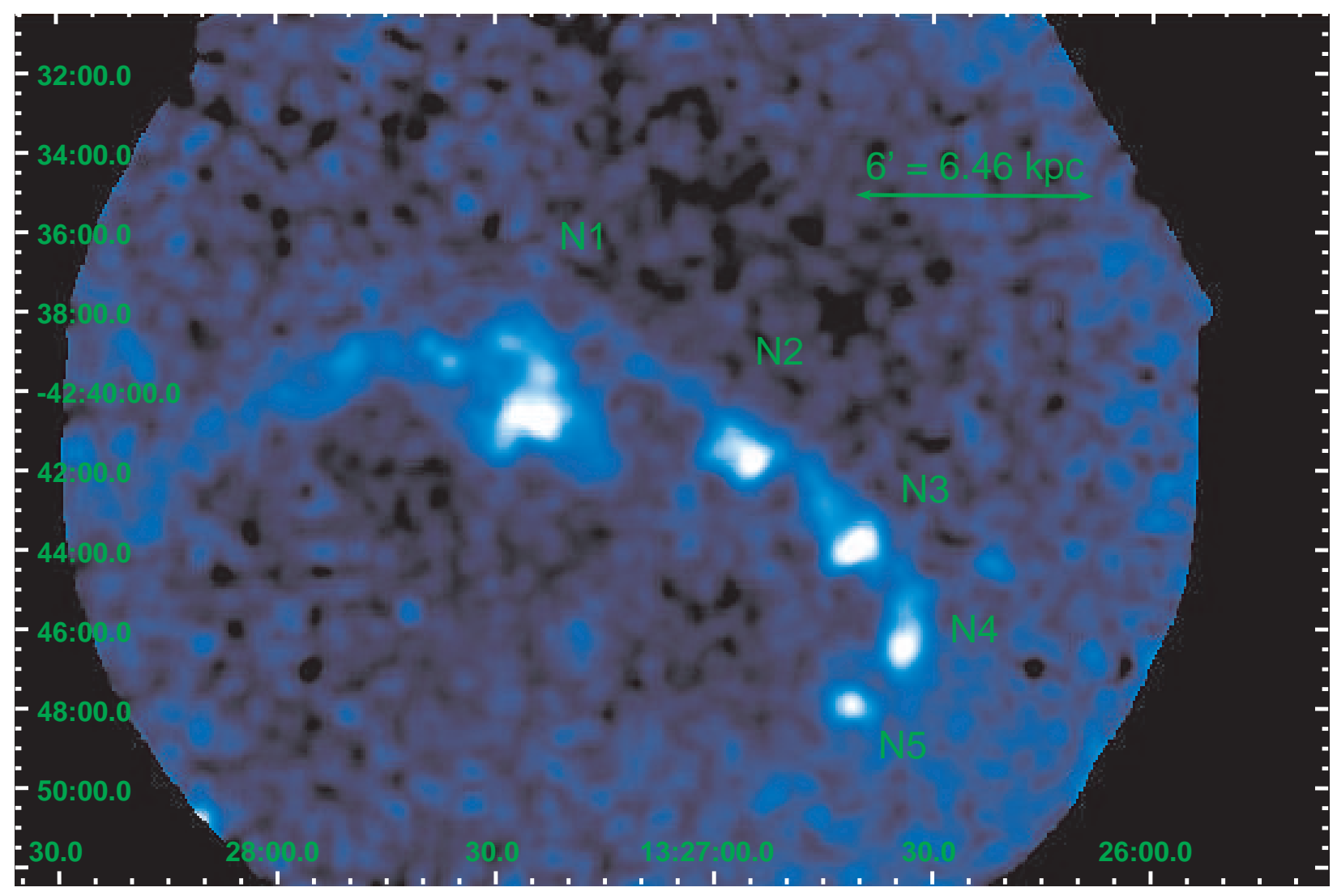

Fig. 4.- Smoothed (Gaussian kernel - $\sigma=24^{\prime \prime}$ ), exposure corrected XMM-Newton MOS1+MOS2 image of the Cen A NML in the $0.5-2.0 \mathrm{keV}$ band with point sources removed. 


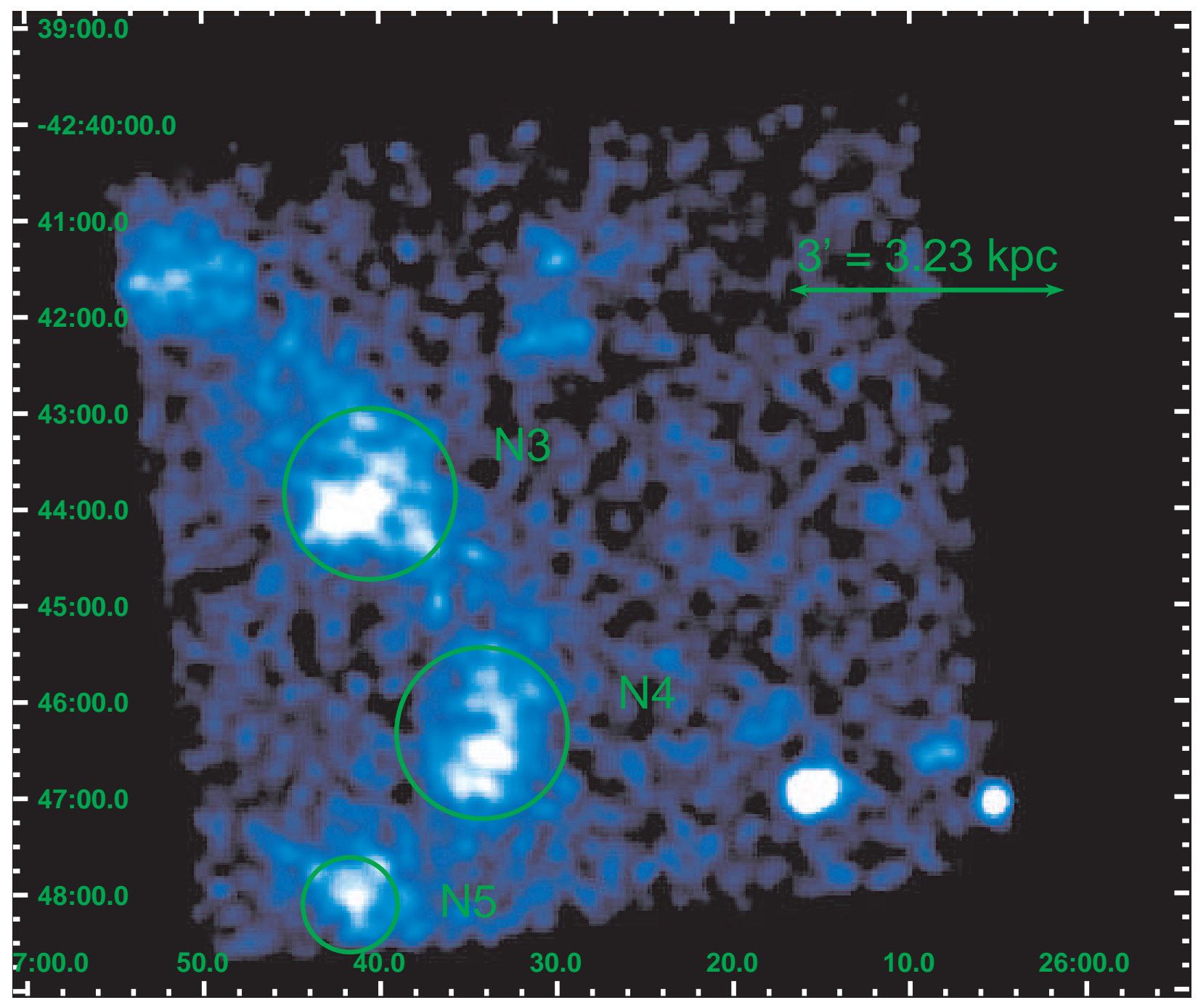

Fig. 5.- Chandra image of knots N3, N4, and N5 of the Cen A NML in the 0.5-2.0 keV bandpass. This region lies roughly $\sim 15^{\prime}$ from best focus. Two point sources to the west of the filament can be used to visually estimate the PSF. All the knots are clearly extend. 


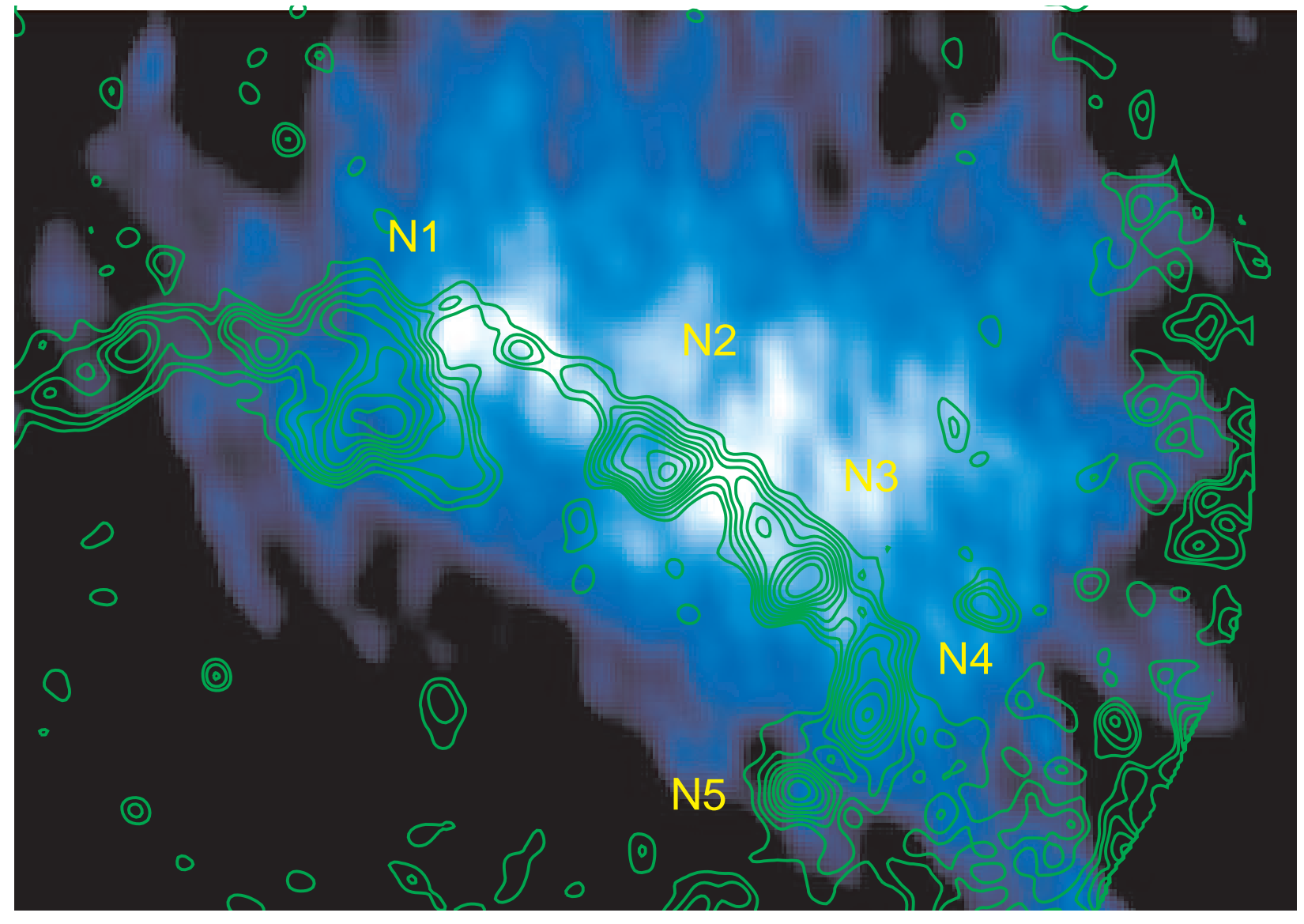

Fig. 6.- Radio map of Cen A NML (1.34 GHz, 60" $(\mathrm{NS}) \times 36^{\prime \prime}(\mathrm{EW})$ beam) with X-ray contours from Figure 4 overlaid. Note the anti-coincidence between the X-ray and radio peaks around knots N1, N2, and N3. 


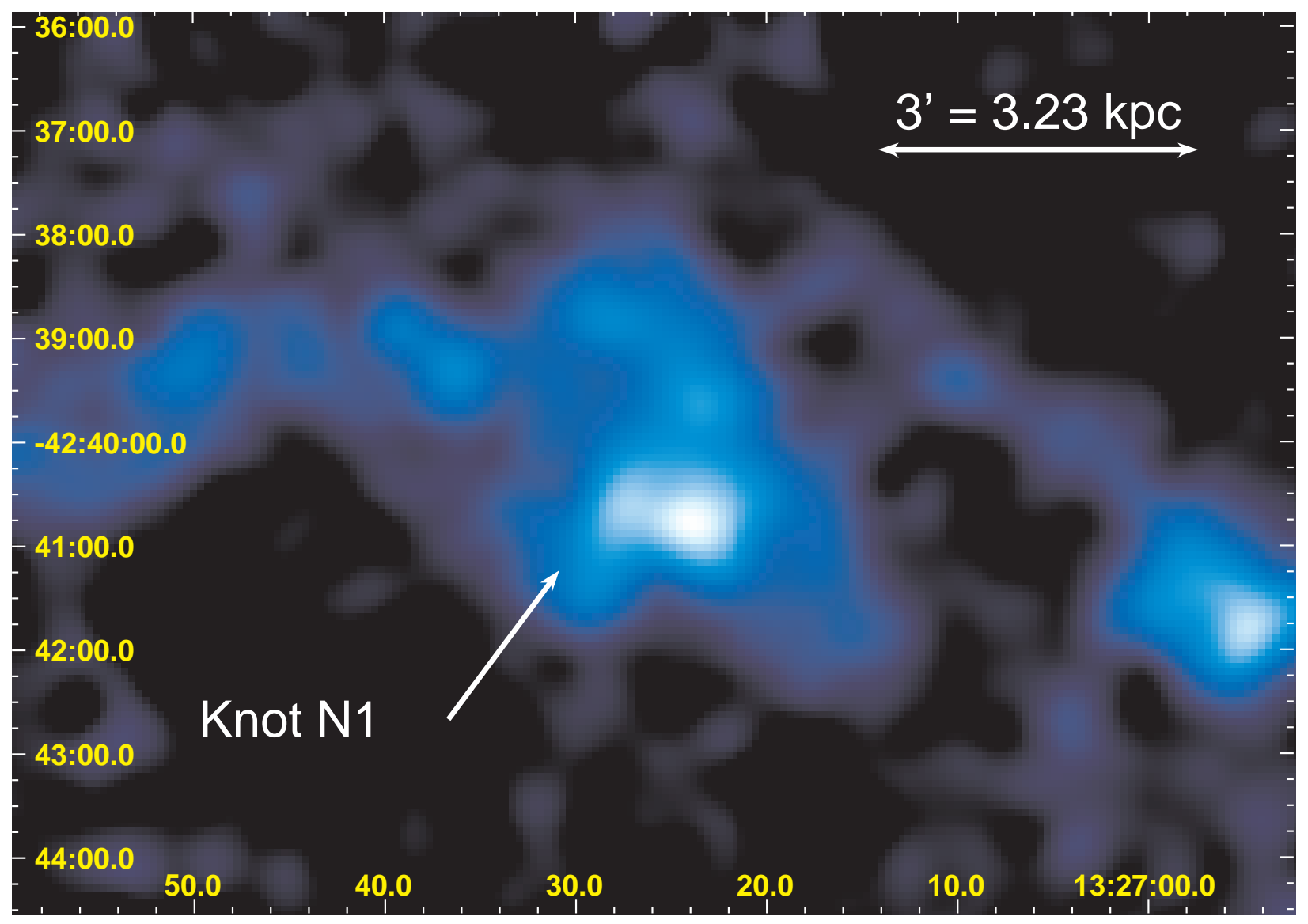

Fig. 7.- XMM-Newton MOS1+MOS2 image (exposure corrected with point sources removed) of knot N1 of the Cen A NML in the 0.5-2.0 keV band. 


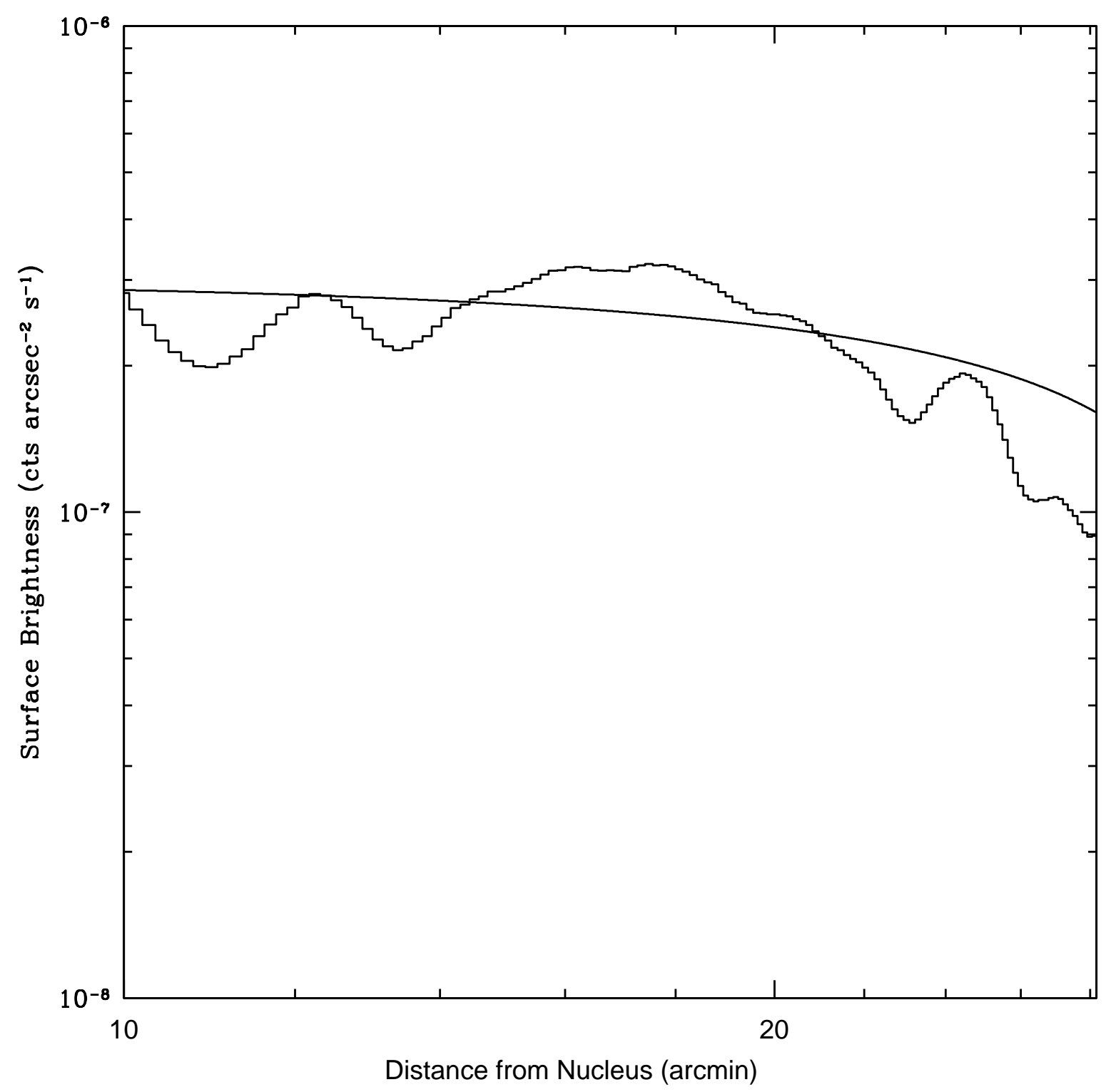

Fig. 8.- Surface brightness profile (histogram) of hot gas in a $10^{\circ}$ wedge $(0.3-1.0 \mathrm{keV})$ southeast of the X-ray filament with vertex at the nucleus. This profile was created from a Gaussian smoothed $\left(\sigma=30^{\prime}\right.$ exposure corrected MOS1+MOS2 image. The continuous curve shows the projected surface brightness of the uniform density sphere discussed in the text. The background level $\left(3.6-4.0 \times 10^{-7} \operatorname{cts~s}^{-1} \operatorname{arcsec}^{-2}\right.$ was determined in a region to the northeast of the X-ray filament. 


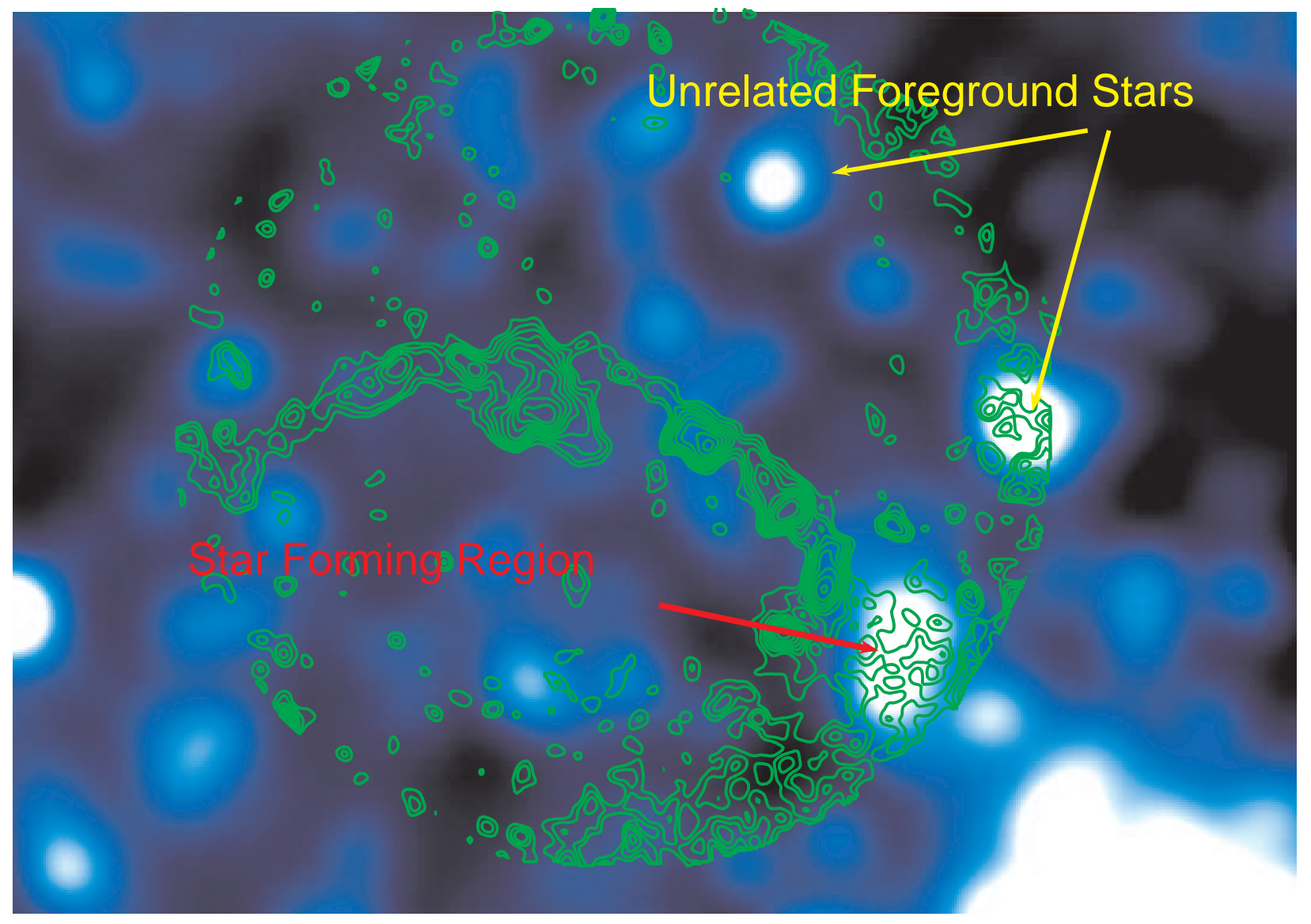

Fig. 9.- XMM-Newton contours (from Figure 4) overlaid onto a GALEX NUV image. The point sources and all other features on spatial scales smaller than 30" arcseconds have been removed via wavelet decomposition. Diffuse emission from the well studied star forming region at the base of the NML is shown with the red arrow. Two bright foreground stars are also noted. 


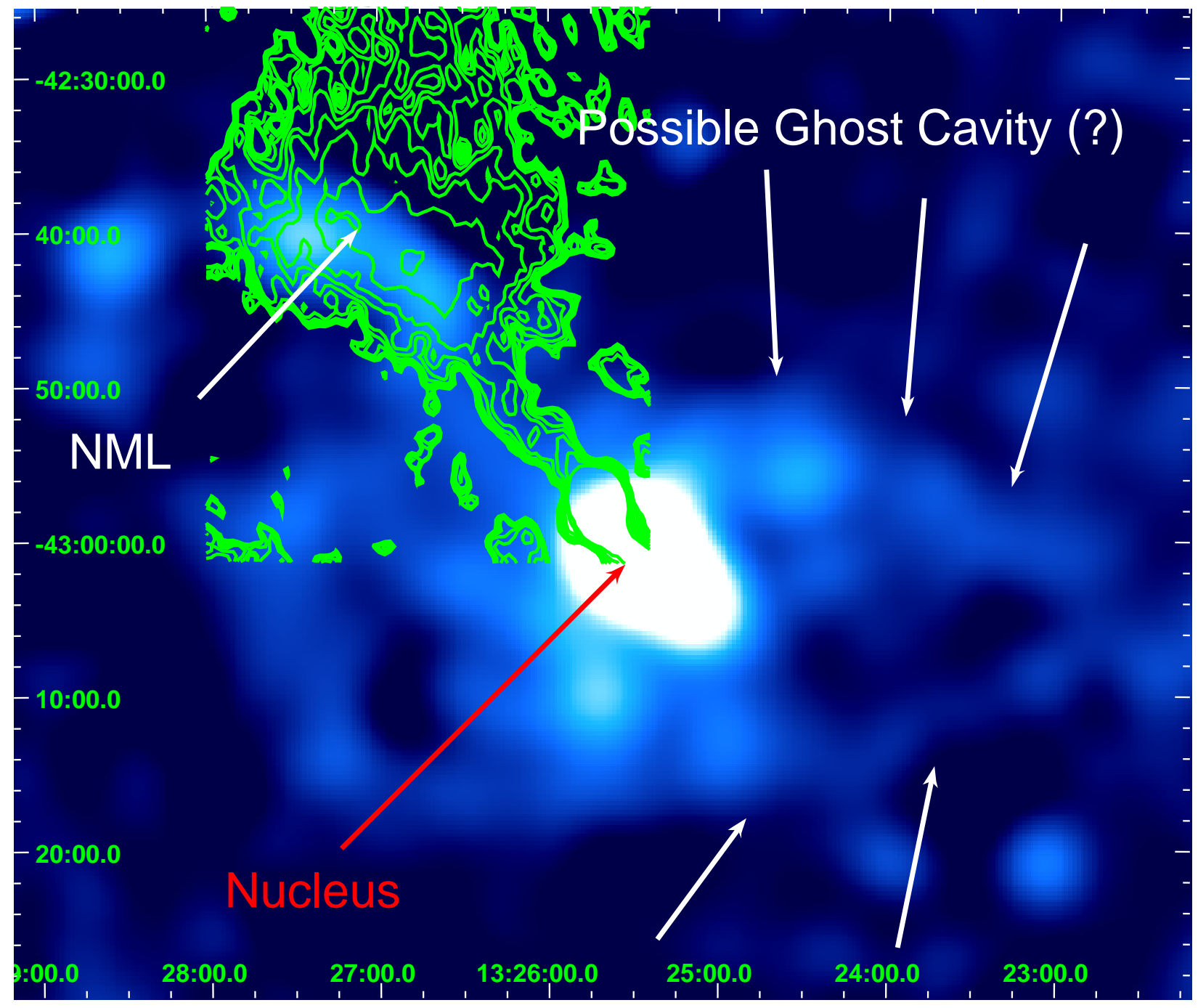

Fig. 10. - Exposure corrected ROSAT PSPC observation (14 ks) of Cen A in the 0.5-1.1 $\mathrm{keV}$ bandpass. The radio contours of the NML are overlaid, and the possible 'ghost' cavity is denoted by the white arrows. 


\begin{tabular}{|c|c|c|c|c|c|c|c|}
\hline Region & RA & Dec & Radius & Temperature $(\mathrm{keV})$ & $Z\left(\times 10^{-2}\right)$ & $L_{X}$ & $\operatorname{Norm}\left(\times 10^{-5}\right)$ \\
\hline N1 & $13: 27: 25.3$ & $-42: 40: 17.5$ & $1.48^{\prime}$ & $0.71 \pm 0.02$ & $9 \pm 2$ & 5.1 & 21. \\
\hline $\mathrm{N} 2$ & $13: 26: 56.8$ & $-42: 41: 37.4$ & $0.98^{\prime}$ & $0.50 \pm 0.04$ & $5 \pm 2$ & 1.8 & 7.5 \\
\hline N3 & $13: 26: 40.5$ & $-42: 43: 50.6$ & $0.89^{\prime}$ & $0.58 \pm 0.07$ & $9 \pm 2$ & 2.0 & 8.1 \\
\hline & & & & $0.65 \pm 0.05$ & $13 \pm 8$ & & \\
\hline N4 & $13: 26: 34.2$ & $-42: 46: 19.8$ & $0.98^{\prime}$ & $0.39 \pm 0.03$ & $5 \pm 3$ & 1.8 & 10. \\
\hline & & & & $0.49 \pm 0.08$ & $<0.2$ & & \\
\hline N5 & $13: 26: 41.7$ & $-42: 48: 06.6$ & $0.30^{\prime}$ & $1.07 \pm 0.02$ & $<0.1$ & 1.0 & 8.3 \\
\hline & & & & $4.1_{1.5}^{4.7}$ & $<1.0$ & & \\
\hline
\end{tabular}

Table 1: Best fit temperatures and uncertainties (90\% confidence) for five knots of the Xray filament of the Cen A NML. The regions with the $C$ suffix are fits to the Chandra data. Uncertainties are $90 \%$ confidence for one parameter of interest, and the coordinates are J2000. The second set of temperatures and elemental abundances for N3, N4, and N5 are from the Chandra data. The $L_{X}$ column is the X-ray luminosity of the knot in the $0.5-2.0 \mathrm{keV}$ band (unabsorbed) in units of $10^{38} \mathrm{ergs} \mathrm{s}^{-1}$. The norm column contains the XSPEC normalization for the spectral fit for $Z=0.5$ (fixed) which are used to derive the densities, pressure, energies, and masses of the knots shown in Table 2. These are not the normalizations for the best-fit, low abundance spectra. 


\begin{tabular}{|c|c|c|c|c|c|}
\hline Region & $\begin{array}{c}\text { Density }\left(n_{H}\right) \\
\left(10^{-2} \mathrm{~cm}^{-3}\right)\end{array}$ & $\begin{array}{c}\text { Pressure } \\
\left(10^{-11} \mathrm{dyn} \mathrm{cm}^{-2}\right)\end{array}$ & $\begin{array}{c}\text { Mass } \\
\left(10^{6} \mathrm{M}_{\odot}\right)\end{array}$ & $\begin{array}{c}\text { Energy } \\
\left(10^{54} \mathrm{ergs}\right)\end{array}$ & $\begin{array}{c}\text { Lifetime } \\
(\mathrm{Myrs})\end{array}$ \\
\hline \hline $\mathrm{N} 1$ & 0.8 & 2.2 & 3.5 & 16.5 & 3.7 \\
\hline $\mathrm{N} 2$ & 1.1 & 2.0 & 1.0 & 3.2 & 2.6 \\
\hline $\mathrm{N} 3$ & 1.1 & 2.4 & 1.0 & 3.8 & 2.4 \\
\hline $\mathrm{N} 4$ & 1.2 & 1.6 & 1.1 & 2.6 & 3.1 \\
\hline $\mathrm{N} 5$ & 2.7 & 23.8 & 0.4 & 6.7 & 0.6 \\
\hline \hline Lobe & & 0.12 & & 300 & \\
\hline
\end{tabular}

Table 2: Thermodynamic parameters of the knots in the Cen A NML X-ray filament assuming $Z=0.5$. The XSPEC normalizations from Table 1 have been scaled to $Z=0.5$ thus reducing the gas density, pressure, and total mass as described in the text. The values listed as pressure and energy for the lobe are the equipartition pressure and the bubble enthalpy (for $\gamma=4 / 3$ assuming equipartition) of the lobe. 